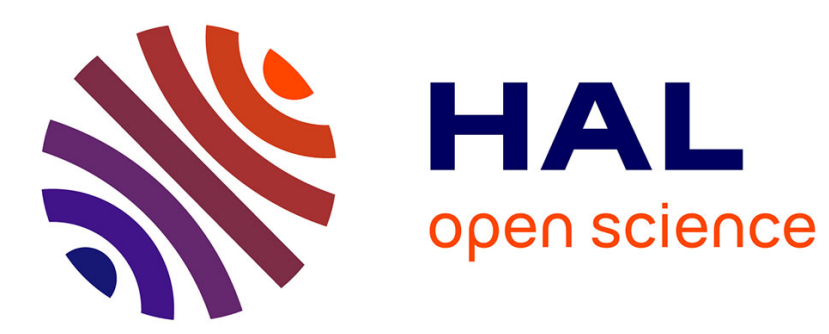

\title{
Decidable Topologies for Communicating Automata with FIFO and Bag Channels
}

Lorenzo Clemente, Frédéric Herbreteau, Grégoire Sutre

\section{To cite this version:}

Lorenzo Clemente, Frédéric Herbreteau, Grégoire Sutre. Decidable Topologies for Communicating Automata with FIFO and Bag Channels. CONCUR 2014 - 25th International Conference on Concurrency Theory, Sep 2014, Rome, Italy. pp.281-296, 10.1007/978-3-662-44584-6_20 . hal-01023456

\section{HAL Id: hal-01023456 https://hal.science/hal-01023456}

Submitted on 13 Jul 2014

HAL is a multi-disciplinary open access archive for the deposit and dissemination of scientific research documents, whether they are published or not. The documents may come from teaching and research institutions in France or abroad, or from public or private research centers.
L'archive ouverte pluridisciplinaire HAL, est destinée au dépôt et à la diffusion de documents scientifiques de niveau recherche, publiés ou non, émanant des établissements d'enseignement et de recherche français ou étrangers, des laboratoires publics ou privés. 


\title{
Decidable Topologies for Communicating Automata with FIFO and Bag Channels ${ }^{\star}$
}

\author{
Lorenzo Clemente ${ }^{1}$, Frédéric Herbreteau ${ }^{2}$, and Grégoire Sutre ${ }^{2}$ \\ 1 Université Libre de Bruxelles, Brussels, Belgium \\ 2 Univ. Bordeaux \& CNRS, LaBRI, UMR 5800, Talence, France
}

\begin{abstract}
We study the reachability problem for networks of finite-state automata communicating over unbounded perfect channels. We consider communication topologies comprising both ordinary FIFO channels and bag channels, i.e., channels where messages can be freely reordered. It is well-known that when only FIFO channels are considered, the reachability problem is decidable if, and only if, there is no undirected cycle in the topology. On the other side, when only bag channels are allowed, the reachability problem is decidable for any topology by a simple reduction to Petri nets. In this paper, we study the more complex case where the topology contains both FIFO and bag channels, and we provide a complete characterisation of the decidable topologies in this generalised setting.
\end{abstract}

\section{Introduction}

Communicating finite-state automata (CFSA) are a fundamental model of computation where concurrent processes exchange messages over unbounded, reliable channels. Depending on the context, messages are delivered in the order they were sent (FIFO channel), or in any order (bag channel). On the one hand, FIFO channels can be used, e.g., to model communications through TCP sockets, as TCP preserves the order of messages. It is well-known that the reachability problem for CFSA with only FIFO channels is undecidable $[5,18]$. This problem becomes decidable when the communication topology is required to be acyclic $[18,14]$. Many other decidable subclasses and under/over-approximation techniques have been considered in the literature $[3,2,7,6,14,8,4,11,10,1]$. On the other hand, bag channels can be used, e.g., to model asynchronous procedure calls $[19,12,9]$. Indeed, libraries supporting asynchronous programming do not guarantee, in general, that procedures are executed in the order they were asynchronously called. The reachability problem for CFSA with only bag channels is decidable (without any further restriction), by an immediate reduction to reachability in Petri nets, which is known to be decidable $[16,13,15]$.

Contributions. While the reachability problem is well-understood for communication topologies of just FIFO or bag channels, we go one step forward and we study topologies comprising both FIFO and bag channels. Our main result is

\footnotetext{
^ This work was partially supported by the ANR project VACSIM (ANR-11-INSE-004).
} 


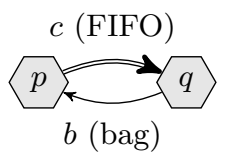

(a) Opposite channels

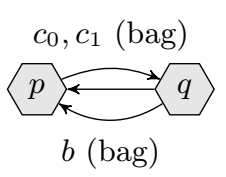

(b) Synchronising $c$

Fig. 1: Opposite channels

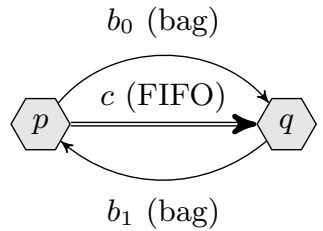

Fig. 2: Undecidable topology

a complete characterisation of decidable topologies of FIFO and bag channels, and a detailed complexity analysis in the decidable case. As a consequence of our results, we show that certain non-trivial cycles comprising FIFO and bag channels can be allowed while preserving decidability.

In addition to being the right model in some contexts, bag channels also provide a non-trivial over-approximation of FIFO channels. Indeed, it is always possible to over-approximate the reachability set by turning all channels into bag channels. Thanks to our characterisation, a much finer analysis may be obtained, in practice, by selectively over-approximating only some of the FIFO channels.

Preview. Let us illustrate our main techniques with some example. While the topology in Fig. 1a is undecidable when all channels are FIFO, it becomes decidable when $b$ is bag. Indeed, the FIFO channel $c$ can be "made synchronous" by forcing receptions to occur right after transmissions. This, in turn, can be implemented by replacing $c$ with two opposite bag channels $c_{0}, c_{1}$ implementing a simple rendezvous protocol. We thus obtain the topology in Fig. 1b, which is decidable since it contains only bag channels.

A more difficult case is the one in Fig. 3a. As above, reachability is undecidable if all channels are FIFO, but it becomes decidable when one channel, say $b$, is bag. However, the correctness argument is more involved here, since, unlike in the previous example, channel $c$ cannot be made synchronous. The problem is that making $c$ synchronous requires rescheduling the actions of the receiver $q$ to occur earlier. But this is not possible since $q$ might try to read on the other channel $b$, which could be empty. The crucial observation is that we can always schedule all actions of $p$ to occur before all actions of $q$. Therefore, in this topology, the order between transmissions and receptions can be relaxed. The only property that matters is that the string of messages which is received is the same as the one which is sent. We can thus split the bag channel $b$ into two bag channels $b_{0}$ and $b_{1}$ (see Fig. 3b), where $q$ 's potentially blocking receptions on $b$ are replaced with non-blocking transmissions on $b_{1}$. The new process $r$ just matches incoming messages on $b_{0}$ and $b_{1}$. In the new topology, $c$ can be made synchronous, and we proceed as above to obtain the decidable topology in Fig. 3c.

Finally, we also have undecidable topologies which mix in a non-trivial way FIFO and bag channels. For example, consider the one in Fig. 2, where $c$ is FIFO and $b_{0}$ and $b_{1}$ are bags. This topology is undecidable, even when $b_{0}$ and $b_{1}$ are unary bag channels (i.e., the message alphabet is a singleton). The idea is to use 


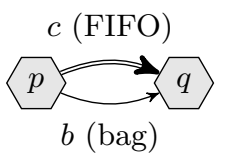

(a) Parallel channels $b$ and $c$

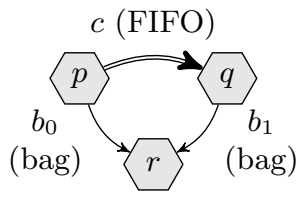

(b) Splitting $b$

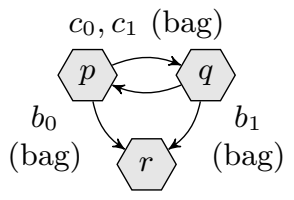

(c) Synchronising $c$

Fig. 3: Two parallel channels

the two bag channels $b_{0}$ and $b_{1}$ to implement a synchronisation protocol between processes $p$ and $q$. This protocol is then used by $p$ to decide which message is to be received by $q$ from $c$, thus simulating a channel machine (which has undecidable reachability).

Outline. The rest of the paper is organised as follows. We start with preliminaries in Sec. 2. In Sec. 3 we show techniques for synchronising and splitting channels (decidability). In Sec. 4 we explain how unary bag channels can be used to simulate rendezvous synchronisation (undecidability). In Sec. 5 we present our characterisation of decidable topologies and study the complexity of the decidable instances. Finally, in Sec. 6 we compare our techniques with the work of Chambart and Schnoebelen [8], and in Sec. 7 we end with directions for future work. Full proofs can be found in the appendix.

\section{Preliminaries}

A labelled transition system (LTS for short) is a tuple $\mathcal{A}=\left\langle S, S_{I}, S_{F}, A, \rightarrow\right\rangle$ where $S$ is a set of states with initial states $S_{I} \subseteq S$ and final states $S_{F} \subseteq S$, $A$ is a finite set of actions, and $\rightarrow \subseteq S \times A \times S$ is a labelled transition relation. For simplicity, we write $s \stackrel{a}{\longrightarrow} s^{\prime}$ in place of $\left(s, a, s^{\prime}\right) \in \rightarrow$. An LTS is called finite when its set of states is finite. A run in $\mathcal{A}$ is an alternating sequence $\left(s_{0}, a_{1}, s_{1}, \ldots, a_{n}, s_{n}\right)$ of states $s_{i} \in S$ and actions $a_{i} \in A$, with $n \geq 0$, such that $s_{i-1} \stackrel{a_{i}}{\longrightarrow} s_{i}$ for all $1 \leq i \leq n$. The natural number $n$, which may be zero, is called the length of the run. An accepting run is a run starting in an initial state (i.e., with $s_{0} \in S_{I}$ ) and ending in a final state (i.e., with $s_{n} \in S_{F}$ ). Given two runs $\sigma=\left(s_{0}, a_{1}, s_{1}, \ldots, a_{m}, s_{m}\right)$ and $\tau=\left(t_{0}, b_{1}, t_{1}, \ldots, b_{n}, t_{n}\right)$ such that $s_{m}=t_{0}$, their join is the run $\sigma \cdot \tau=\left(s_{0}, a_{1}, s_{1}, \ldots, a_{m}, s_{m}, b_{1}, t_{1}, \ldots, b_{n}, t_{n}\right)$.

Topologies. We consider systems that are composed of several processes communicating through the asynchronous exchange of messages. Communications rely on point-to-point FIFO channels between processes. In our setting, each channel is equipped with a message alphabet that specifies the set of messages that can be conveyed over the channel. To simplify the presentation, we assume a special message, written 1, that is always in the message alphabet. Formally, a communication topology is a tuple $\mathcal{T}=\langle P, C, M$, src, dst, msg $\rangle$, where $P$ is a finite set of 
processes, $C$ is a finite set of channels, $M$ is a finite set of messages containing the special message 1, src : $C \rightarrow P$ and dst : $C \rightarrow P$ are mappings assigning to each channel a source and a destination process, and msg :C $\rightarrow\{N \subseteq M \mid 1 \in N\}$ is a mapping assigning to each channel its message alphabet. For convenience, we assume that the sets $P, C$ and $M$ are pairwise disjoint. A channel $c \in C$ is called unary when $\operatorname{msg}(c)$ is a singleton, i.e., when $\operatorname{msg}(c)=\{1\}$.

The following graph-theoretic concepts and notations ${ }^{3}$ will be used throughout the paper. Consider a topology $\mathcal{T}=\langle P, C, M$, src, dst, msg $\rangle$. For each channel $c \in C$, we let $\stackrel{c}{\Longrightarrow}$ denote the binary relation on $P$ defined by $p \stackrel{c}{\Longrightarrow} q$ if $p=\operatorname{src}(c)$ and $q=\operatorname{dst}(c)$. The inverse of $\stackrel{c}{\Longrightarrow}$ is written $\stackrel{c}{=}$. A directed walk in $\mathcal{T}$ is an alternating sequence $\left(p_{0}, c_{1}, p_{1}, \ldots, c_{n}, p_{n}\right)$ of processes $p_{i} \in P$ and channels $c_{i} \in C$, with $n \geq 0$, such that $p_{i-1} \stackrel{c_{i}}{\Longrightarrow} p_{i}$ for all $1 \leq i \leq n$. The natural number $n$, which may be zero, is called the length of the directed walk. To improve readability, directed walks will usually be written $p_{0} \stackrel{c_{1}}{\Longrightarrow} p_{1} \cdots \stackrel{c_{n}}{\Longrightarrow} p_{n}$. A directed walk is said to be closed when it starts and ends in the same process (i.e., when $p_{0}=p_{n}$ ). A directed path is a directed walk in which all channels are pairwise distinct, and all processes - except, possibly, the first and last ones - are pairwise distinct. The notation $p \stackrel{*}{\Longrightarrow} q$ means that there is a directed walk - or, equivalently, there is a directed path - from $p$ to $q$ (i.e., with $p_{0}=p$ and $p_{n}=q$ ). A directed cycle is a closed directed path of non-zero length.

We also need undirected variants of the above notions. For each channel $c \in C$, we let $\stackrel{c}{=}$ denote the binary relation on $P$ defined by $p \stackrel{c}{=} q$ if $\{p, q\}=$ $\{\operatorname{src}(c), \operatorname{dst}(c)\}$. Observe that $p \stackrel{c}{=} q$ if, and only if, $p \stackrel{c}{\Longrightarrow} q$ or $p \stackrel{c}{\Longleftarrow} q$. The notions of undirected walk, undirected path and undirected cycle are defined as the directed ones, except that $\stackrel{c}{\Longrightarrow}$ is replaced by $\stackrel{c}{=}$.

Communicating processes. Given a topology $\mathcal{T}=\langle P, C, M$, src, dst, msg $\rangle$, the set of possible communication actions for a process $p \in P$, written $A_{\mathrm{com}}^{p}$, is the union of the set $\{c ! m \mid c \in C \wedge \operatorname{src}(c)=p \wedge m \in \operatorname{msg}(c)\}$ of its transmission actions and of the set $\{c ? m \mid c \in C \wedge \operatorname{dst}(c)=p \wedge m \in \operatorname{msg}(c)\}$ of its reception actions. The set of all communication actions is $A_{\text {com }}=\bigcup_{p \in P} A_{\text {com }}^{p}$. Actions not in $A_{\text {com }}$ are called internal actions.

Definition 2.1. A system of communicating processes is a pair $\mathcal{S}=\left\langle\mathcal{T},\left\{\mathcal{A}^{p}\right\}_{p \in P}\right\rangle$ where $\mathcal{T}=\langle P, \ldots\rangle$ is a topology, and, for each $p \in P, \mathcal{A}^{p}=\left\langle S^{p}, S_{I}^{p}, S_{F}^{p}, A^{p}, \rightarrow^{p}\right\rangle$ is a finite labelled transition system such that $A^{p} \cap A_{\mathrm{com}}=A_{\mathrm{com}}^{p}$. For convenience, we assume $e^{4}$ that the sets of actions $A^{p}$, with $p \in P$, are pairwise disjoint.

We give the operational semantics of a system of communicating processes $\mathcal{S}$ as a global labelled transition system $\llbracket \mathcal{S} \rrbracket=\left\langle X, X_{I}, X_{F}, A, \rightarrow\right\rangle$. States of $\mathcal{S}$ are called configurations to prevent confusion with those of $\mathcal{A}^{p}$. A configuration

\footnotetext{
${ }^{3}$ In this paper, we use contemporary graph terminology (see, e.g., [20]). For instance, the term walk is used for "paths" that may repeat channels and/or processes.

${ }^{4}$ This assumption is not restrictive as it only concerns internal actions. Indeed, the sets $A_{\mathrm{com}}^{p}$, with $p \in P$, are already pairwise disjoint by definition.
} 
of $\llbracket \mathcal{S} \rrbracket$ is pair $x=(\boldsymbol{s}, \boldsymbol{w})$ where $s$ maps each process $p$ to a state in $S^{p}$, and $\boldsymbol{w}$ maps each channel $c$ to a word over its message alphabet msg $(c)$. Formally, $X=\left(\prod_{p \in P} S^{p}\right) \times\left(\prod_{c \in C} \mathrm{msg}(c)^{*}\right)$. A configuration is initial (resp. final) when each process is in its initial state (resp. final state) and all channels are empty. Formally, $X_{I}=\left(\prod_{p \in P} S_{I}^{p}\right) \times\{\varepsilon\}$ and $X_{F}=\left(\prod_{p \in P} S_{F}^{p}\right) \times\{\varepsilon\}$, where $\varepsilon$ maps each channel $c \in C$ to the empty word $\varepsilon$. The set of actions $A$ of $\mathcal{S}$ is given by $A=\bigcup_{p \in P} A^{p}$. Observe that $\left\{A^{p}\right\}_{p \in P}$ is a partition of $A$. We define the transition relation $\rightarrow$ of $\llbracket \mathcal{S} \rrbracket$ to be the set of all triples $\left(x_{1}, a, x_{2}\right)$, where $x_{1}=\left(\boldsymbol{s}_{1}, \boldsymbol{w}_{1}\right)$ and $x_{2}=\left(\boldsymbol{s}_{2}, \boldsymbol{w}_{2}\right)$ are configurations, such that, for some process $p \in P$, the following conditions are satisfied:

$-s_{1}^{p} \stackrel{a}{\longrightarrow} s_{2}^{p}$ is a transition in $\mathcal{A}^{p}$, and $s_{1}^{q}=s_{2}^{q}$ for all other processes $q \in P \backslash\{p\}$.

- If $a$ is an internal action, then $\boldsymbol{w}_{1}=\boldsymbol{w}_{2}$.

- If $a=c ! m$, then $w_{2}^{c}=w_{1}^{c} \cdot m$ and $w_{2}^{d}=w_{1}^{d}$ for all other channels $d \in C \backslash\{c\}$.

- If $a=c ? m$, then $w_{1}^{c}=m \cdot w_{2}^{c}$ and $w_{2}^{d}=w_{1}^{d}$ for all other channels $d \in C \backslash\{c\}$.

So, in a transition $x_{1} \stackrel{a}{\longrightarrow} x_{2}$, exactly one process $p$ moves (namely, the unique $p \in P$ such that $a \in A^{p}$ ), and the others stay put. The channels are updated according to the action $a$ that is performed by the transition. Given a process $p \in P$, a move of $p$ is any transition $x_{1} \stackrel{a}{\longrightarrow} x_{2}$ such that $a \in A^{p}$. Following [11], we define the causal-equivalence relation $\sim$ over runs as the least congruence, with respect to join, such that $\left(x_{1}, a, x_{2}, b, x_{3}\right) \sim\left(x_{1}, b, x_{2}^{\prime}, a, x_{3}\right)$ whenever $a, b$ are actions of distinct processes. Informally, two runs are causal-equivalent if they can be transformed one into the other by iteratively commuting adjacent moves that (i) are not from the same process and (ii) do not form a "matching send/receive pair". It is readily seen that causal-equivalent runs necessarily start in the same configuration and end in the same configuration.

Statement of the problem. Given a topology $\mathcal{T}$, the reachability problem for systems of communicating processes with topology $\mathcal{T}$, denoted by $\operatorname{REACH}(\mathcal{T})$, is defined as follows:

Input: a system of communicating processes $\mathcal{S}$ with topology $\mathcal{T}$, Output: whether there exists an accepting run in $\llbracket \mathcal{S} \rrbracket$.

Observe that we require all channels to be empty at the end of an accepting run. Also note that $\operatorname{REACH}(\mathcal{T})$ is parametrised by a topology $\mathcal{T}$. The main result of this paper is a characterisation of the topologies $\mathcal{T}$ for which $\operatorname{REACH}(\mathcal{T})$ is decidable. Our techniques are based on topological transformations that induce reductions between the associated reachability problems. We let $\preceq_{m}$ denote the many-one reducibility relation between decision problems. For example, $\operatorname{REACH}(\mathcal{T}) \preceq_{m} \operatorname{REACH}(\mathcal{U})$ when $\mathcal{T}$ is obtained from $\mathcal{U}$ by removing channels.

When only unary channels are present, the reachability problem is decidable by an immediate reduction to reachability in Petri nets.

Theorem $2.2([16,13,15])$. If $\mathcal{T}$ is a topology with only unary channels, then $\operatorname{REACH}(\mathcal{T})$ is decidable. 
On the other hand, when the topology contains only non-unary channels the following characterisation for $\operatorname{REACH}(\mathcal{T})$ is well-known.

Theorem 2.3 ([18,14]). Given a topology $\mathcal{T}$ with no unary channel, $\operatorname{REACH}(\mathcal{T})$ is decidable if, and only if, $\mathcal{T}$ has no undirected cycle.

In Sec. 5, we refine the latter condition to account for topologies with both unary and non-unary channels (see Theorem 5.3). We further generalise it in Sec. 6 to a more general setting comprising both FIFO and bag channels.

\section{Synchronising and Splitting Channels}

A useful technique in the analysis of communicating processes is to transform asynchronous communications into synchronous ones, without compromising the behaviour of the system. To this end, we use the notion of synchronous runs from $[8,11]$. Formally, a run $\left(\left(\boldsymbol{s}_{0}, w_{0}\right), a_{1},\left(\boldsymbol{s}_{1}, w_{1}\right), \ldots, a_{n},\left(\boldsymbol{s}_{n}, w_{n}\right)\right)$ is synchronous for a given channel $c$ if $w_{0}^{c}=w_{n}^{c}=\varepsilon$ and $w_{i}^{c}=\varepsilon \vee w_{i+1}^{c}=\varepsilon$ for all $0<i<n$. Intuitively, this means that each transmission on $c$ is immediately followed by its matching reception, i.e., communication over $c$ behaves like rendezvous synchronisation [17]. It is well-known that in a polyforest topology (i.e., with no undirected cycle), every run can be reordered to have all channels synchronous, and reachability can be solved by exploring the resulting finite transition system $[18,14,8,11]$. Since we are interested in analysing more complex topologies where not all channels can be simultaneously made synchronous in general, we need to consider channels individually rather than globally.

Synchronising essential channels. Whether a channel can always be made synchronous (by reordering moves in runs) is a semantic condition that depends on the complex behaviour of the whole system. In fact, this condition is an undecidable problem in general (by an easy reduction from the reachability problem). Therefore, we are interested in syntactic conditions that are sufficient for a channel to be made synchronous. One such condition is that of essential channel [8], which is a structural condition depending only on the topology.

Definition 3.1 ([8]). A channel $c$ is essential if all directed paths from $\operatorname{src}(c)$ to $\operatorname{dst}(c)$ contain $c$. In particular, $\operatorname{src}(c) \neq \mathrm{dst}(c)$.

Lemma $3.2([8])$. If $c$ is an essential channel, then every run that starts and ends with c empty is causal-equivalent to a run that is synchronous for $c$.

Thus, we can replace asynchronous communications on an essential channel by synchronous ones. We loosely implement the latter through a topological transformation that replaces an essential channel $c$, with source $p$ and destination $q$, by one unary channel $c_{m}$ from $p$ to $q$ for each message $m \in \operatorname{msg}(c)$, as well as one unary channel $c_{\text {ack }}$ back from $q$ to $p$. These unary channels are enough to simulate synchronous communications over $c$. Each message $m$ conveyed over $c$ is placed in the corresponding unary channel $c_{m}$ instead. After each transmission on 
$c_{m}$, the process $p$ waits for an acknowledgement from $c_{\text {ack }}$. Conversely, $q$ notifies $p$ via $c_{\text {ack }}$ after each reception from $c_{m}$. While it applies to arbitrary essential channels, this topological transformation is only useful for non-unary ones.

Definition 3.3. Given a topology $\mathcal{T}=\langle P, C, M$, src, dst, msg $\rangle$ and a channel $c \in C$, the synchronisation of $c$ in $\mathcal{T}$ is the topology $\mathcal{U}=\left\langle P, C^{\prime}, M, \mathrm{src}^{\prime}, \mathrm{dst}^{\prime}, \mathrm{msg}^{\prime}\right\rangle$ defined by $C^{\prime}=(C \backslash\{c\}) \cup\left\{c_{m} \mid m \in \operatorname{msg}(c)\right\} \cup\left\{c_{\text {ack }}\right\}$ and

$$
\left(\operatorname{src}^{\prime}(d), \operatorname{dst}^{\prime}(d), \operatorname{msg}^{\prime}(d)\right)= \begin{cases}(\operatorname{src}(d), \operatorname{dst}(d), \operatorname{msg}(d)) & \text { if } d \in C \backslash\{c\} \\ (\operatorname{src}(c), \operatorname{dst}(c),\{1\}) & \text { if } d=c_{m} \\ (\operatorname{dst}(c), \operatorname{src}(c),\{1\}) & \text { if } d=c_{\text {ack }}\end{cases}
$$

where $c_{m}$, for $m \in \operatorname{msg}(c)$, and $c_{\text {ack }}$ are new channels that are not in $P \cup C \cup M$.

Proposition 3.4 (Synchronisation). If $c$ is an essential channel in $\mathcal{T}$, then $\operatorname{REACH}(\mathcal{T}) \preceq_{m} \operatorname{REACH}(\mathcal{U})$ where $\mathcal{U}$ results from the synchronisation of $c$ in $\mathcal{T}$.

Remark 3.5. An essential channel could, alternatively, be removed by merging its endpoints (see [8]). Instead, our synchronisation construction replaces an essential channel by a collection of new unary channels. While either technique could be used for decidability (see Subsec. 5.2), synchronisation yields simpler proofs and avoids taking the product of LTSes at the process level, which cirvumvents an immediate exponential blow-up in our reduction to reachability in Petri nets (see Subsec. 5.3). From a practical viewpoint, the new unary channels are 1-bounded by construction; analyzers for Petri nets could take advantage of this fact.

Splitting irreversible channels. According to Proposition 3.4 above, a topology containing an essential non-unary channel $c$ can always be simplified by synchronising $c$. The resulting topology is simpler in the sense that it contains one less non-unary channel. However, there are situations where a channel is not essential, and thus it cannot be synchronised in general, but it can be made essential after a small modification. We have shown one such simple case on Fig. 3a, where the channel $c$ is not essential but it can be made so by splitting ${ }^{5}$ the other channel $p \stackrel{b}{\Longrightarrow} q$ into two channels $p \stackrel{b_{0}}{\Longrightarrow} r$ and $q \stackrel{b_{1}}{\Longrightarrow} r$ for a new process $r$, see Fig. 3b. Here, $r$ is a new process that simply matches messages received from $b_{0}$ and $b_{1}$; receptions on $b$ become transmissions on $b_{1}$. Clearly, the new system with the split topology has at least the same runs as the original system. Moreover, in this case, the converse holds as well, since we can always schedule all actions of $p$ before any action of $q$, thus causality between transmissions and receptions can be relaxed. Formally, the splitting operation is defined as follows.

Definition 3.6. Given a topology $\mathcal{T}=\langle P, C, M$, src, dst, msg $\rangle$ and a channel $c \in C$, the split of $c$ in $\mathcal{T}$ is the topology $\mathcal{U}=\left\langle P^{\prime}, C^{\prime}, M\right.$, $\left.\mathrm{src}^{\prime}, \mathrm{dst}^{\prime}, \mathrm{msg}^{\prime}\right\rangle$ defined

\footnotetext{
${ }^{5}$ Despite having similar names, this splitting notion and the splitting technique of [8] have little in common (see Sec. 6).
} 
by $P^{\prime}=P \cup\{r\}, C^{\prime}=(C \backslash\{c\}) \cup\left\{c_{0}, c_{1}\right\}$, and

$$
\left(\operatorname{src}^{\prime}(d), \mathrm{dst}^{\prime}(d), \mathrm{msg}^{\prime}(d)\right)= \begin{cases}(\operatorname{src}(d), \operatorname{dst}(d), \operatorname{msg}(d)) & \text { if } d \in C \backslash\{c\} \\ (\operatorname{src}(c), r, \operatorname{msg}(c)) & \text { if } d=c_{0} \\ (\operatorname{dst}(c), r, \operatorname{msg}(c)) & \text { if } d=c_{1}\end{cases}
$$

where $r$ is a new process and $c_{0}, c_{1}$ are new channels that are not in $P \cup C \cup M$.

To justify splitting, we introduce the notion of causal run. Intuitively, in a run which is causal for a process $p$, only those processes that can "transitively" send messages to $p$ may be scheduled before $p$.

Definition 3.7. A run $\left(x_{0}, a_{1}, x_{1}, \ldots, a_{n}, x_{n}\right)$ is causal for a given process $p$ if $q \stackrel{*}{\Longrightarrow} p$ for every process $q$ such that $a_{i} \in A^{q}$ and $a_{j} \in A^{p}$ for some $1 \leq i<j \leq n$.

Lemma 3.8. Given a process $p$, every run is causal-equivalent to a run that is causal for $p$.

Recall that the idea behind splitting is to relax the causality between transmissions and receptions. This does not introduce "spurious" runs provided that every run can be reordered to have all actions of the receiver after the last action of the sender. A sufficient condition is given by the notion of irreversible channel.

Definition 3.9. A channel $c$ is reversible if there is a directed path from its destination $\operatorname{dst}(c)$ to its source $\operatorname{src}(c)$. A channel is irreversible if it is not reversible.

The following proposition states that the reachability problem for a given topology $\mathcal{T}$ can be reduced to the reachability problem for the topology obtained from $\mathcal{T}$ by splitting $c$. As we will see in Sec. 5 , splitting will be the first of a series of reductions for decidable topologies.

Proposition 3.10 (Split). If $c$ is an irreversible channel in $\mathcal{T}$, then it holds that $\operatorname{REACH}(\mathcal{T}) \preceq_{m} \operatorname{REACH}(\mathcal{U})$ where $\mathcal{U}$ results from the split of $c$ in $\mathcal{T}$.

\section{The Power of Unary Channels}

Let $u$ be a process in a topology $\mathcal{T}$, and let $\mathcal{U}$ be a topology which is the same as $\mathcal{T}$ except that $u$ is expanded into a strongly-connected sub-topology. In this section, we show that the behaviour of $u$ in $\mathcal{T}$ can be distributed over its expansion in $\mathcal{U}$. We achieve this by demonstrating how processes in a stronglyconnected sub-topology can simulate global rendezvous synchronisation over a finite alphabet of shared actions, which allows them to synchronise with each others and to step-wise simulate the behaviour of $u$. This technique works even when distributing behaviour over unary channels only, and it will be used to show the undecidability part of our characterisation (see Subsec. 5.1).

To formally define how a process is expanded into a sub-topology, it is more convenient to describe how to fuse a set of channels $D$. Intuitively, the fusion of $D$ in a given topology is the topology obtained by merging together, in a single process, all endpoints of channels in $D$, by removing $D$, and by redirecting the other channels in the natural way. 

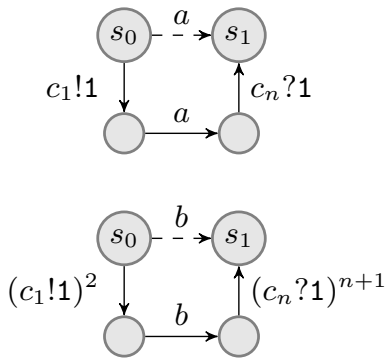

(a) Process $p_{0}$
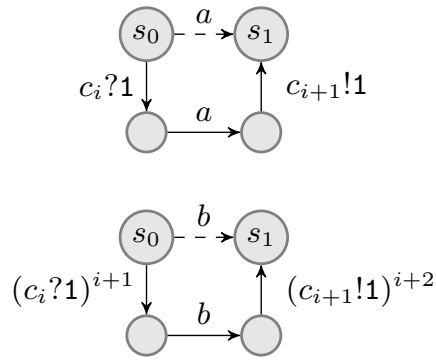

(b) Process $p_{i} \neq p_{0}$

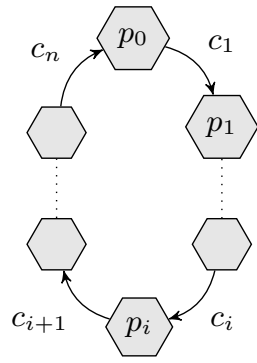

(c) Topology

Fig. 4: Synchronisation protocol for a simple directed cycle

Definition 4.1. Given a topology $\mathcal{T}=\langle P, C, M$, src, dst, msg $\rangle$ and a set of channels $D \subseteq C$, the fusion of $D$ in $\mathcal{T}$ is the topology $\mathcal{U}=\left\langle P^{\prime}, C^{\prime}, M\right.$, $\left.\mathrm{src}^{\prime}, \mathrm{dst}^{\prime}, \mathrm{msg}^{\prime}\right\rangle$ defined by $P^{\prime}=(P \backslash\{\operatorname{src}(c)$, dst $(c) \mid c \in D\}) \cup\{u\}$ where $u$ is a new process that is not in $P \cup C \cup M, C^{\prime}=C \backslash D$, and, for every $c \in C^{\prime}, \mathrm{msg}^{\prime}(c)=\operatorname{msg}(c)$, $\operatorname{src}^{\prime}(c)=\operatorname{src}(c)$ if $\operatorname{src}(c) \in P^{\prime}$ and $\operatorname{src}^{\prime}(c)=u$ otherwise, and similarly for $\mathrm{dst}^{\prime}$.

This section shows that fusing a strongly-connected sub-topology makes the reachability problem easier. We first deal with the simple case of directed cycles. The support of a directed walk/cycle is the set of channels that it visits.

Lemma 4.2. If $D$ is the support of a directed cycle in a topology $\mathcal{T}$ then $\operatorname{REACH}(\mathcal{U}) \preceq_{m} \operatorname{REACH}(\mathcal{T})$ where $\mathcal{U}$ results from the fusion of $D$ in $\mathcal{T}$.

Proof. Consider a directed cycle $p_{0} \stackrel{c_{1}}{\Longrightarrow} p_{1} \stackrel{c_{2}}{\Longrightarrow} \cdots \stackrel{c_{n}}{\Longrightarrow} p_{n}=p_{0}$ in $\mathcal{T}$ such that $D=\left\{c_{1}, \ldots, c_{n}\right\}$. This directed cycle is depicted in Fig. 4c. Denote by $\mathcal{U}$ the topology that results from the fusion of $D$ in $\mathcal{T}$, and let $u$ be the process in $\mathcal{U}$ that corresponds to the merging of all endpoints of $D$. Consider a system of communicating processes $\mathcal{S}$ with topology $\mathcal{U}$. We construct a new system $\mathcal{R}$ with topology $\mathcal{T}$ that simulates $\mathcal{S}$ by "distributing" the behaviour of the process $u$ over $p_{1}, \ldots, p_{n}$. All other processes are left unchanged.

As a first step, let us assume that the processes $p_{1}, \ldots, p_{n}$ can perform multiway rendezvous synchronisation over a finite alphabet of actions $\Sigma$. By multi-way, we mean that each time some process $p_{i}$ performs an action $a$ in $\Sigma$, then in fact all processes $p_{1}, \ldots, p_{n}$ perform the action $a$ at the same time. For brevity, we will omit the "multi-way" qualifier from now on. It is easily shown (see App. B for details) that rendezvous synchronisation, even over a binary alphabet, allows $p_{1}, \ldots, p_{n}$ to coordinate in $\mathcal{T}$ and simulate the behaviour of $u$ in $\mathcal{U}$. This way, we obtain from $\mathcal{S}$ a new system $\mathcal{S}^{\prime}$, with topology $\mathcal{T}$, such that $\llbracket \mathcal{S} \rrbracket$ has an accepting run if, and only if, $\llbracket \mathcal{S}^{\prime} \rrbracket$ does. Moreover, $\mathcal{S}^{\prime}$ does not use any channel in $D$.

As a second step, we explain how rendezvous synchronisation between $p_{1}, \ldots, p_{n}$ over a binary alphabet, say $\{a, b\}$, can be achieved through communications on the channels in $D$. In our simulation, rendezvous synchronisations are initiated by $p_{0}$, and then propagated along the directed cycle $p_{0} \stackrel{c_{1}}{\Longrightarrow} p_{1} \stackrel{c_{2}}{\Longrightarrow} \cdots \stackrel{c_{n}}{\Longrightarrow} p_{n}$ back 
to $p_{0}$. The latter then checks that the other processes correctly performed the desired rendezvous action. More precisely, whenever $p_{0}$ wants to handshake on some action $\left(a\right.$ or $b$ ), it sends some number of messages on $c_{1}$ to $p_{1}$ and waits for an acknowledgement on $c_{n}$ before proceeding to its next move. In the meantime, the processes $p_{1}, \ldots, p_{n-1}$ do the same, but first receive and then transmit. The number of messages received by $p_{i+1}$ from $c_{i+1}$ is exactly the same as the number of messages sent by $p_{i}$ on $c_{i+1}$. The precise protocol is shown in Fig. 4, where each dashed transitions in $\mathcal{S}^{\prime}$ is replaced in $\mathcal{R}$ by the alternative sequence below it (by introducing intermediate states). All other transitions are left unchanged. In $\mathcal{R}$, actions $a$ and $b$ are to be interpreted as internal, non-rendezvous actions. For instance, to simulate the rendezvous action $a, p_{0}$ first sends a message on $c_{1}$, internally performs $a$, and then receives a message from $c_{n}$.

To conclude the proof, we show that $\llbracket \mathcal{S}^{\prime} \rrbracket$ has an accepting run if, and only if, $\llbracket \mathcal{R} \rrbracket$ does. By construction, each rendezvous synchronisation in $\llbracket \mathcal{S}^{\prime} \rrbracket$ can be reproduced, through the above protocol, in $\llbracket \mathcal{R} \rrbracket$. We now argue that the protocol does not introduce any spurious behaviour. Recall that the channels $c_{1}, \ldots, c_{n}$ are empty at the beginning. So, for each $1 \leq i \leq n-1$, the process $p_{i}$ may only simulate a rendezvous action after $p_{0}$ has initiated a synchronisation round. Let us look at the first rendezvous action that is simulated by $p_{0}$. If this action is $a$, then $p_{0}$ sends one message on $c_{1}$ and receives one message from $c_{n}$. This entails that all the other processes $p_{1}, \ldots, p_{n-1}$ simulate the rendezvous action $a$. At the end of this synchronisation round, all the channels $c_{1}, \ldots, c_{n}$ are again empty. If the first rendezvous action that $p_{0}$ simulates is $b$, then $p_{0}$ sends two messages on $c_{1}$ and receives $n+1$ messages from $c_{n}$. Again, this entails that $p_{1}, \ldots, p_{n-1}$ simulate, each, the rendezvous action $b$. Indeed, by contradiction, if $p_{i}$ simulates $a$, then it must continue simulating $a$ since there are not enough messages in $c_{i}$ anymore to simulate $b$. Therefore, it simply relays messages from $c_{i}$ to $c_{i+1}$, and cannot produce on $c_{i+1}$ the extra message that $p_{i+1}$ expects to simulate $b$. By applying the same arguments to the remaining processes $p_{i+1}, \ldots, p_{n-1}$, we obtain that $p_{n-1}$ is not able to produce on $c_{n}$ the $n+1$ messages that $p_{0}$ expects to complete its simulation of $b$, a contradiction. Again, at the end of this synchronisation round, all the channels $c_{1}, \ldots, c_{n}$ are empty. By repeating this analysis for each synchronisation round, we obtain that every accepting run of $\llbracket \mathcal{R} \rrbracket$ can be mapped back to an accepting run of $\llbracket \mathcal{S}^{\prime} \rrbracket$.

We now show that the previous lemma still holds for closed directed walks, i.e., where processes/channels can be repeated. The proof is by induction on the cardinality of $D$. As expected, the induction step follows from Lemma 4.2.

Proposition 4.3 (Fusion). If $D$ is the support of a closed directed walk in a topology $\mathcal{T}$, then $\operatorname{REACH}(\mathcal{U}) \preceq_{m} \operatorname{REACH}(\mathcal{T})$ where $\mathcal{U}$ results from the fusion of $D$ in $\mathcal{T}$.

\section{Characterisation of Decidable Topologies}

We are now ready to state and prove our characterisation of decidable topologies. The characterisation is expressed in the same vein as Theorem 2.3, and generalises 
it. We first introduce some additional definitions and notations. Consider a topology $\mathcal{T}=\langle P, C, M$, src, dst, msg $\rangle$, and let $D \subseteq C$ be a subset of channels. Two processes $p$ and $q$ are synchronizable over $D$, written $p \approx_{D} q$, if there exists a directed path from $p$ to $q$ and a directed path from $q$ to $p$, both using only channels in $D$. Note that $\approx_{D}$ is an equivalence relation on $P$.

A jumping circuit is a sequence $\left(p_{0}, c_{1}, q_{1}, p_{1}, \ldots, c_{n}, q_{n}, p_{n}\right)$ of processes $p_{i}, q_{i} \in P$ and channels $c_{i} \in C$, with $n \geq 1$, such that $c_{1}, \ldots, c_{n}$ are pairwise distinct non-unary channels, $p_{0}=p_{n}$, and $p_{i-1} \stackrel{c_{i}}{=} q_{i} \approx_{D} p_{i}$ for all $1 \leq i \leq n$, where $D=C \backslash\left\{c_{1}, \ldots, c_{n}\right\}$. Recall that the binary relation $\stackrel{c}{=}$ is the union of $\stackrel{c}{\Longrightarrow}$ and $\stackrel{c}{\Longleftarrow}$. A jumping cycle is a jumping circuit $\left(p_{0}, c_{1}, q_{1}, p_{1}, \ldots, c_{n}, q_{n}, p_{n}\right)$ such that $q_{i} \varkappa_{D} q_{j}$ for all $1 \leq i<j \leq n$. To improve readability, jumping circuits and jumping cycles will often be written $p_{0} \stackrel{c_{1}}{=} q_{1} \approx_{D} p_{1} \ldots \stackrel{c_{n}}{=} q_{n} \approx_{D} p_{n}$.

Remark 5.1. Every jumping circuit can be transformed into a jumping cycle.

Remark 5.2. For every jumping cycle $p_{0} \stackrel{c_{1}}{=} q_{1} \approx_{D} p_{1} \ldots \stackrel{c_{n}}{=} q_{n} \approx_{D} p_{n}$, there exist $n$ pairwise disjoint subsets $D_{1}, \ldots, D_{n}$ of the set $D=C \backslash\left\{c_{1}, \ldots, c_{n}\right\}$ such that $q_{i} \approx_{D_{i}} p_{i}$ for all $1 \leq i \leq n$.

Theorem 5.3. Given a topology $\mathcal{T}, \operatorname{REACH}(\mathcal{T})$ is decidable if, and only if, $\mathcal{T}$ has no jumping cycle.

The two directions of the theorem are proved in the two subsections below. To illustrate our characterisation, let us give some examples of decidable topologies. Certainly, polyforest topologies are decidable since they contain no undirected cycle, therefore no jumping cycle. Moreover, decidability is preserved if we add, for each channel of the polyforest, a unary channel in the opposite direction (see Lemma 5.4 below). Even further, we still get a decidable topology if each process is expanded into a sub-topology containing only unary channels. These operations introduce non-trivial cycles of unary and non-unary channels. Finally, adding unary channels looping on the same process always preserves decidability, as well as adding additional unary channels in parallel to already existing ones.

\section{$5.1 \quad$ Undecidability}

Consider a topology $\mathcal{T}$ containing a jumping cycle $\xi=\left(p_{0}, c_{1}, q_{1}, p_{1}, \ldots, c_{n}, q_{n}, p_{n}\right)$. By Remark 5.2, it holds that $p_{0} \stackrel{c_{1}}{=} q_{1} \approx_{D_{1}} p_{1} \ldots \stackrel{c_{n}}{=} q_{n} \approx_{D_{n}} p_{n}$ for some pairwise disjoint subsets $D_{1}, \ldots, D_{n}$ of $C \backslash\left\{c_{1}, \ldots, c_{n}\right\}$. We may assume, w.l.o.g., that each $D_{i}$ is the support of a closed directed walk in $\mathcal{T}$. To prove that $\operatorname{REACH}(\mathcal{T})$ is undecidable, we show that $\operatorname{REACH}(\mathcal{U}) \preceq_{m} \operatorname{REACH}(\mathcal{T})$ for some topology $\mathcal{U}$ with an undirected cycle containing only non-unary channels, hence, for which reachability is undecidable by Theorem 2.3. To do so, we build a sequence of topologies $\mathcal{U}_{0}, \mathcal{U}_{1}, \ldots, \mathcal{U}_{n}$ by fusing together synchronizable processes, as follows: We define $\mathcal{U}_{0}=\mathcal{T}$, and, for each $1 \leq i \leq n$, we let $\mathcal{U}_{i}$ result from the fusion of $D_{i}$ in $\mathcal{U}_{i-1}$. Pairwise disjointness of $D_{1}, \ldots, D_{n}$ ensures that every channel in $D_{i}$ is still a channel in $\mathcal{U}_{i-1}$. It is routinely checked (see Appendix C.1 for details) that: 
- $D_{i}$ is still the support of a closed directed walk in $\mathcal{U}_{i-1}$, and

- $\xi$ induces a closed undirected walk $u_{0} \stackrel{c_{1}}{=} u_{1} \ldots \stackrel{c_{n}}{=} u_{n}$ in $\mathcal{U}_{n}$.

The first item entails, by Proposition 4.3, that $\operatorname{REACH}\left(\mathcal{U}_{i}\right) \preceq_{m} \operatorname{REACH}\left(\mathcal{U}_{i-1}\right)$. By the transitivity of $\preceq_{m}$, we get that $\operatorname{REACH}\left(\mathcal{U}_{n}\right) \preceq_{m} \operatorname{REACH}\left(\mathcal{U}_{0}\right)$. Since $c_{1}, \ldots, c_{n}$ are pairwise distinct non-unary channels, we derive, from the second item, that $\mathcal{U}_{n}$ contains an undirected cycle with only non-unary channels. It follows from Theorem 2.3 that $\operatorname{REACH}\left(\mathcal{U}_{n}\right)$ is undecidable. As $\mathcal{U}_{0}=\mathcal{T}$, we conclude that $\operatorname{REACH}(\mathcal{T})$ is undecidable.

\subsection{Decidability}

Starting from a topology $\mathcal{T}$ with no jumping cycle, we apply a sequence of topological transformations that produce a topology $\mathcal{U}$ with only unary channels, and such that $\operatorname{REACH}(\mathcal{T}) \preceq_{m} \operatorname{REACH}(\mathcal{U})$. Since the latter is decidable by Theorem 2.2, the former is decidable as well.

Given a topology $\mathcal{T}$ and a channel $c$ in $\mathcal{T}$, an acknowledgement channel for $c$ is a new unary channel, written $\overleftarrow{c}$, with source $\operatorname{dst}(c)$ and destination $\operatorname{src}(c)$. The following lemma says that adding an acknowledgement channel for an essential non-unary channel preserves the absence of jumping cycle. It immediately entails Corollary 5.5 below, and its full proof can be found in App. C.2.

Lemma 5.4. Consider a topology $\mathcal{T}$ and an essential non-unary channel $c$ therein. Let $\mathcal{U}$ be the topology obtained from $\mathcal{T}$ by adding an acknowledgement channel for $c$. Then $\mathcal{T}$ contains a jumping cycle if $\mathcal{U}$ contains a jumping cycle.

Proof (sketch). Assume that $\mathcal{T}$ has no jumping cycle, but adding $\overleftarrow{c}$ yields a topology $\mathcal{U}$ with a jumping cycle $p_{0} \stackrel{c_{1}}{=} q_{1} \approx_{D_{1}} p_{1} \ldots \stackrel{c_{n}}{=} q_{n} \approx_{D_{n}} p_{n}=p_{0}$. Observe that $\overleftarrow{c}$ cannot be one of $c_{1}, \ldots, c_{n}$ since it is unary. If $\overleftarrow{c}$ does not appear in any $D_{i}$, then $\mathcal{T}$ has a jumping cycle. Hence, $\overleftarrow{c}$ appears on a closed directed walk $\pi$ that synchronises two processes $q_{i}$ and $p_{i}$. Since $c$ is essential, it must appear on $\pi$ too. We may assume, w.l.o.g., that $\overleftarrow{c}$ appears on the directed path from $p_{i}$ to $q_{i}$ on $\pi$, and that $c$ appears on the directed path from $q_{i}$ to $p_{i}$. We get that $q_{i} \stackrel{*}{\Longrightarrow} p \stackrel{c}{\Longrightarrow} q \stackrel{*}{\Longrightarrow} p_{i} \stackrel{*}{\Longrightarrow} q \stackrel{\overleftarrow{c}}{\Longrightarrow} p \stackrel{*}{\Longrightarrow} q_{i}$. Therefore, $q_{i}$ can synchronise with $p$ using only channel from $E_{i}=D_{i} \backslash\{c, \overleftarrow{c}\}$, and similarly $p_{i}$ can synchronise with $q$ via $E_{i}$. So we can build a jumping cycle in $\mathcal{T}$ from the jumping cycle in $\mathcal{U}$ by replacing $q_{i} \approx_{D_{i}} p_{i}$ by $q_{i} \approx_{E_{i}} p \stackrel{c}{\Longrightarrow} q \approx_{E_{i}} p_{i}$, a contradiction.

Corollary 5.5. Consider a topology $\mathcal{T}$ and an essential non-unary channel $c$ therein. Let $\mathcal{U}$ be the topology resulting from the synchronisation of $c$ in $\mathcal{T}$. Then $\mathcal{T}$ contains a jumping cycle if $\mathcal{U}$ contains a jumping cycle.

Remark 5.6. The converse of Corollary 5.5 also holds, but it is not required for the proof of Theorem 5.3.

We say that a topology $\mathcal{T}$ is divided if the destination of every irreversible unary channel is a sink (i.e., is not the source of some channel) and is not the destination of some non-unary channel. The following two properties of divided topologies are crucial in the proof of Theorem 5.3. 
Lemma 5.7. Consider a topology $\mathcal{T}$ and a non-unary channel $c$ therein. If $\mathcal{T}$ is divided, then so is the topology resulting from the synchronisation of $c$ in $\mathcal{T}$.

Lemma 5.8. If $\mathcal{T}$ is a divided topology with no jumping cycle, then every nonunary channel in $\mathcal{T}$ is essential.

We now prove the "if" direction of Theorem 5.3. Assume that $\mathcal{T}$ has no jumping cycle. Let $c_{1}, \ldots, c_{n}$ denote the non-unary channels of $\mathcal{T}$. We first build $\mathcal{U}_{0}$ from $\mathcal{T}$ by splitting all unary channels that are irreversible in $\mathcal{T}$. Note that $\mathcal{U}_{0}$ does not depend on the order in which the irreversible unary channels of $\mathcal{T}$ are split. It follows from Proposition 3.10 and the transitivity of $\preceq_{m}$ that $\operatorname{REACH}(\mathcal{T}) \preceq_{m} \operatorname{REACH}\left(\mathcal{U}_{0}\right)$. By construction, the topology $\mathcal{U}_{0}$ is divided, and it still has no jumping cycle. So, by Lemma 5.8, every non-unary channel in $\mathcal{U}_{0}$ is essential. Notice that $\mathcal{U}_{0}$ has the same non-unary channels as $\mathcal{T}$, namely $c_{1}, \ldots, c_{n}$. For each $1 \leq i \leq n$, let $\mathcal{U}_{i}$ be the topology resulting from the synchronisation of $c_{i}$ in $\mathcal{U}_{i-1}$. By induction, it is immediate to prove that, for every $0 \leq i \leq n$, $\mathcal{U}_{i}$ has no jumping cycle, the induction step holding by Corollary 5.5, that $\mathcal{U}_{i}$ is divided, by Lemma 5.7 , that $c_{i+1}, \ldots, c_{n}$ are still essential in $\mathcal{U}_{i}$, by Lemma 5.8 , and that $\operatorname{REACH}\left(\mathcal{U}_{i-1}\right) \preceq_{m} \operatorname{REACH}\left(\mathcal{U}_{i}\right)$, by Proposition 3.4. By the transitivity of $\preceq_{m}$, we get that $\operatorname{REACH}(\mathcal{T}) \preceq_{m} \operatorname{REACH}\left(\mathcal{U}_{n}\right)$. Since $\mathcal{U}_{n}$ contains only unary channels, $\operatorname{REACH}\left(\mathcal{U}_{n}\right)$ is decidable by Theorem 2.2. $\operatorname{Thus} \operatorname{REACH}(\mathcal{T})$ is decidable.

\subsection{Complexity}

We consider the reachability problem for systems of communicating processes whose topology has no jumping cycle. This problem, written REACHNJC, is the union of the problems $\operatorname{REACH}(\mathcal{T})$ for topologies $\mathcal{T}$ with no jumping cycle. Note that deciding whether a topology has a jumping cycle is a simple graph-theoretic problem that can be solved in polynomial time. Hence, it can be checked efficiently whether a given system of communicating processes is an instance of REACHNJC or not. Here, we show that REACHNJC is equivalent to reachability in Petri nets.

The size of a labelled transition system $\mathcal{A}=\left\langle S, S_{I}, S_{F}, A, \rightarrow\right\rangle$ is defined as $|\mathcal{A}|=|S|+|\rightarrow|$. Similarly, the size of a topology $\mathcal{T}=\langle P, C, M$, src, dst, msg $\rangle$ is $|\mathcal{T}|=|P|+|C|+\sum_{c \in C}|\mathrm{msg}(c)|$. Finally, the size of a system of communicating processes $\mathcal{S}=\left\langle\mathcal{T},\left\{\mathcal{A}^{p}\right\}_{p \in P}\right\rangle$ is $|\mathcal{S}|=|\mathcal{T}|+\sum_{p \in P}\left|\mathcal{A}^{p}\right|$. The algorithm in Subsec. 5.2 transforms a system $\mathcal{S}$ over a topology with no jumping cycle, into a system $\mathcal{S}^{\prime}$ with unary channels only. Since unary channels are essentially counters (over the natural numbers) that may only be incremented and decremented, $\mathcal{S}^{\prime}$ can be naturally interpreted as a Petri net. Crucially, we show that $\mathcal{S}^{\prime}$ (and thus the Petri net) has size polynomial in $|\mathcal{S}|$. This is possible since the synchronisation operation from Sec. 3 avoids taking the product of processes (at the cost of introducing 1-bounded unary channels/counters).

Theorem 5.9. ReachNJC is equivalent to reachability in Petri nets under polynomial-time many-one reductions. 


\section{Discussion}

Unary vs. bag channels. A bag channel is a channel where messages can be freely reordered. Therefore, it suffices to count how many messages of each type are in the channel. So, a bag channel over a message alphabet of cardinality $n$ can be implemented with $n$ unary channels in parallel. A topology of bag and FIFO channels is a topology (as defined in Sec. 2) where, in addition, each channel has a flag indicating whether it is ordered (FIFO) or not (bag). Our characterisation from Sec. 5 immediately generalises to bag channels by modifying the definition of jumping cycle and requiring that the $c_{i}$ 's be non-unary FIFO channels (instead of just non-unary).

Unary/bag vs. lossy channels. Another over-approximation incomparable with bag channels is provided by lossy channels, where messages might be lost at any moment $[2,7]$. A complete characterisation of decidable topologies mixing perfect and lossy channels has been presented in [8]. In order to reduce to basic decidable topologies, they consider two reduction rules. The first one is the fusion of essential channels. This is similar in spirit to our synchronisation (see Proposition 3.4), with the only difference that fusing channels requires to take the product of the underlying processes, while synchronising channels just replaces one channel with several 1-bounded unary channels. This allows us to obtain precise complexity bounds in Subsec. 5.3. The second reduction rule is splitting a complex topology $\mathcal{T}$ into $\mathcal{T}_{1}$ and $\mathcal{T}_{2}$ when all channels between $\mathcal{T}_{1}$ and $\mathcal{T}_{2}$ are unidirectional and lossy. Despite similar names, this is different to our splitting technique (see Proposition 3.10), because we split (irreversible) channels, and not topologies. However, while lossy channels cannot be split, the lossy channels involved in the splitting of $\mathcal{T}$ in the sense of [8] are irreversible in our terminology, and thus could be split if they were perfect channels. Moreover, if we replace lossy channels with perfect bag channels, it additionally holds that, if $\mathcal{T}_{1}, \mathcal{T}_{2}$ above are decidable in our characterisation (i.e., no jumping cycles), then the same holds for $\mathcal{T}$. Since also fusion/synchronisation preserves decidable topologies in both settings, we have that any decidable topology of perfect and lossy channels is still a decidable topology by replacing lossy channels with perfect bag channels.

Moreover, some topologies which are undecidable with lossy channels become decidable with bag channels. For example, the topology with three parallel channels $c, d, e$ between two processes with $c$ perfect FIFO and $d, e$ lossy FIFO is undecidable, while if $d, e$ are bag channels it becomes decidable.

Finally, while the topology in Fig. 2 is undecidable when channels $b_{0}$ and $b_{1}$ are either both bag channels or both lossy channels, our construction with unary bag channels is correct even if those are unary and lossy. Indeed, as soon as any message gets lost due to lossiness, our synchronization protocol gets irremediably stuck. However, the construction from [8] does not generalise to unary channels in this case. Thus, we extend their undecidability result to this topology. 


\section{Conclusions and Future Work}

We have presented a complete characterisation of the decidable topologies for networks of finite-state automata communicating over FIFO and bag channels. Remarkably, every decidable topology can be solved using two simple techniques (synchronising essential channels and splitting irreversible channels), whereas every topology that cannot be solved with these two techniques is undecidable.

The same characterisation problem is solved in [8] but for networks mixing perfect and lossy FIFO channels. A direction for future research is to characterise decidable topologies of lossy/perfect FIFO/bag channels.

Relaxing FIFO channels to the bag type can be applied in other contexts as well. For example, the work [11] studies topologies of networks of pushdown automata communicating over FIFO channels, and it is natural to ask what happens when some channels and/or pushdown stores are bags instead of strings.

Acknowledgements. We kindly acknowledge fruitful discussions with Jérôme Leroux, and we thank the anonymous reviewers for helpful comments.

\section{References}

1. Abdulla, P.A., Atig, M.F., Cederberg, J.: Analysis of message passing programs using SMT-solvers. In: Proc. ATVA'13. pp. 272-286 (2013)

2. Abdulla, P., Jonsson, B.: Verifying programs with unreliable channels. Inf. Comput. 127(2), 91-101 (1996)

3. Boigelot, B., Godefroid, P.: Symbolic verification of communication protocols with infinite state spaces using QDDs. Form. Methods Sys. Des. 14, 237-255 (1999)

4. Bouajjani, A., Emmi, M.: Bounded phase analysis of message-passing programs. In: Proc. TACAS'12. pp. 451-465 (2012)

5. Brand, D., Zafiropulo, P.: On communicating finite-state machines. J. ACM 30(2), 323-342 (1983)

6. Cécé, G., Finkel, A.: Verification of programs with half-duplex communication. Inf. Comput. 202(2), 166-190 (2005)

7. Cécé, G., Finkel, A., Purushothaman Iyer, S.: Unreliable channels are easier to verify than perfect channels. Inf. Comput. 124(1), 20-31 (1996)

8. Chambart, P., Schnoebelen, Ph.: Mixing lossy and perfect fifo channels. In: Proc. CONCUR'08. pp. 340-355 (2008)

9. Ganty, P., Majumdar, R.: Algorithmic verification of asynchronous programs. ACM Trans. Program. Lang. Syst. 34(1), 6:1-6:48 (2012)

10. Haase, C., Schmitz, S., Schnoebelen, P.: The power of priority channel systems. In: Proc. CONCUR'13. pp. 319-333 (2013)

11. Heußner, A., Leroux, J., Muscholl, A., Sutre, G.: Reachability analysis of communicating pushdown systems. LMCS 8(3), 1-20 (2012)

12. Jhala, R., Majumdar, R.: Interprocedural analysis of asynchronous programs. SIGPLAN Not. 42(1), 339-350 (2007)

13. Kosaraju, S.R.: Decidability of reachability in vector addition systems (preliminary version). In: Proc. STOC'82. pp. 267-281 (1982)

14. La Torre, S., Madhusudan, P., Parlato, G.: Context-bounded analysis of concurrent queue systems. In: Proc. TACAS'08. pp. 299-314 (2008) 
15. Leroux, J.: Vector addition system reachability problem: a short self-contained proof. In: Proc. POPL'11. pp. 307-316 (2011)

16. Mayr, E.: An algorithm for the general petri net reachability problem. In: Proc. STOC'81. pp. 238-246 (1981)

17. Milner, R.: Communication and Concurrency. Prentice Hall (1989)

18. Pachl, J.K.: Reachability problems for communicating finite state machines. Research Report CS-82-12, University of Waterloo (May 1982)

19. Sen, K., Viswanathan, M.: Model checking multithreaded programs with asynchronous atomic methods. In: Proc. CAV'06. pp. 300-314 (2006)

20. West, D.B.: Introduction to Graph Theory. Prentice Hall, 2 edn. (2001) 


\section{A Proofs for Section 3}

Consider a topology $\mathcal{T}=\langle P, C, M$, src, dst, msg $\rangle$ and a system of communicating processes $\mathcal{S}=\left\langle\mathcal{T},\left\{\mathcal{A}^{p}\right\}_{p \in P}\right\rangle$. We first prove Lemma 3.8 since we use it in the proof of Lemma 3.2. The notion of causal run is formalised in Definition 3.7, while the relation of causal-equivalence is defined in Sec. 2.

Lemma 3.8. Given a process $p$, every run is causal-equivalent to a run that is causal for $p$.

Proof. We show, by induction on the length of runs, that every run $\rho$ satisfies the following property: for every process $p$, there exists a run causal for $p$ that is causal-equivalent to $\rho$. This property obviously holds for runs of length at most one. Consider a run $\rho$ of length two, written as $\rho=t_{p} \cdot t_{q}$, where $t_{p}$ and $t_{q}$ are moves of processes $p$ and $q$, respectively. This run is obviously causal for every process distinct from $q$. If $p \stackrel{*}{\Longrightarrow} q$, then it is also causal for $q$. Otherwise, $p \neq q$ and there is no channel with source $p$ and destination $q$. So the moves of $p$ and $q$ can be swapped in $\rho$, yielding a causal-equivalent run that is causal for $q$.

Consider now a run $\rho$ of length at least three, and let $p$ be a process. If $\rho$ contains no move of $p$, then $\rho$ is trivially causal for $p$. So we assume, for the remainder of the proof, that $\rho$ contains a move of $p$. We first show that $\rho$ is causal-equivalent to a run whose first move is by a process $r$ such that $r \stackrel{*}{\Longrightarrow} p$. The run $\rho$ may be written as $\rho=t \cdot \chi$, where $t$ is the first move of $\rho$ and $\chi$ is a run of length at least two. If $t$ is a move of $p$, then $\rho$ is already in the desired form (since $p \stackrel{*}{\Longrightarrow} p$ ). Otherwise, $\chi$ necessarily contains a move of $p$. According to the induction hypothesis, $\chi$ is causal-equivalent to a run $\chi^{\prime}$ that is causal for $p$. The run $\chi^{\prime}$, whose length is at least two, may be written as $\chi^{\prime}=t_{q} \cdot \chi^{\prime \prime} \cdot t^{\prime}$ where $t_{q}$ is a move of a process $q$ such that $q \stackrel{*}{\Longrightarrow} p$. Notice that $\rho$ is causal-equivalent to $t \cdot t_{q} \cdot \chi^{\prime \prime} \cdot t^{\prime}$. By applying the induction hypothesis to the process $q$ and the run $t \cdot t_{q} \cdot \chi^{\prime \prime}$, we get a run $\rho^{\prime}$ causal for $q$ that is causal-equivalent to $t \cdot t_{q} \cdot \chi^{\prime \prime}$. Observe that $\rho$ is causal-equivalent to $\rho^{\prime} \cdot t^{\prime}$. Moreover, since $\rho^{\prime}$ is causal for $q$ and contains a move of $q$, the first move of $\rho^{\prime}$ is by a process $r$ such that $r \stackrel{*}{\Longrightarrow} q \stackrel{*}{\Longrightarrow} p$. We have shown that $\rho$ is causal-equivalent to a run $t_{r} \cdot \rho^{\prime \prime}$ whose first move $t_{r}$ is by a process $r$ such that $r \stackrel{*}{\Longrightarrow} p$. Replacing $\rho^{\prime \prime}$ by a causal-equivalent run that is causal for $p$ (which is possible by the induction hypothesis) concludes the proof of the lemma.

The proof of Lemma 3.2 given in [8] uses concepts and techniques from the theory of true concurrency. Here, we provide an alternative, more direct proof.

Lemma $3.2([8])$. If $c$ is an essential channel, then every run that starts and ends with c empty is causal-equivalent to a run that is synchronous for $c$.

Proof. By induction on the length of runs. The basis is trivial. Consider a run $\rho$, of non-zero length, that starts and ends with $c$ empty. If the first action of $\rho$ is not a transmission on $c$, then we obtain a causal-equivalent run that is synchronous on $c$ by applying the induction hypothesis to the remainder of $\rho$. Assume, on the 
contrary, that $\rho$ starts with a transmission on $c$. The run $\rho$ may be decomposed into $\rho=x_{1} \stackrel{c ! m}{\longrightarrow} x_{1}^{\prime} \cdot \rho_{1} \cdot x_{2} \stackrel{c ? m}{\longrightarrow} x_{2}^{\prime} \cdot \rho_{2}$ where $\rho_{1}$ contains no reception on $c$.

Let $p$ and $q$ denote the source and target of $c$, respectively. We first show that the run $\chi=x_{1} \stackrel{c ! m}{\longrightarrow} x_{1}^{\prime} \cdot \rho_{1}$ can be reordered into a run of the form $\chi_{1} \cdot y_{1} \stackrel{c ! m}{\longrightarrow} y_{1}^{\prime} \cdot \chi_{2}$ such that $\chi_{1}$ contains no move of $p$ and $\chi_{2}$ contains no move of $q$. Consider the system $\hat{\mathcal{S}}$ obtained from $\mathcal{S}$ by removing the channel $c$ and transforming all communication actions on $c$ into internal actions (written " $c$ ! $m$ " and " $c ? m$ "). Let $\hat{\chi}$ denote the run in $\hat{\mathcal{S}}$ obtained from $\chi$ in the obvious way. Since $c$ is essential in $\mathcal{T}$, there is no directed path from $p$ to $q$ in the topology $\hat{\mathcal{T}}$ of $\hat{\mathcal{S}}$. By Lemma 3.8, the run $\hat{\chi}$ is causal-equivalent to a run that is causal for $q$. Hence, the latter is of the form $\hat{\chi}_{1} \cdot \hat{y}_{1} \stackrel{\text { "c!m" }}{\longrightarrow} \hat{y}_{1}^{\prime} \cdot \hat{\chi}_{2}$ such that $\hat{\chi}_{1}$ contains no move of $p$ and $\hat{\chi}_{2}$ contains no move of $q$. Observe that this run contains no " $c$ ? $m$ ". So it can be mapped back to $\mathcal{S}$, and yields a run that is causal-equivalent to $\chi$ and of the desired form.

The run $\rho$ is causal-equivalent to the run $\chi_{1} \cdot y_{1} \stackrel{c ! m}{\longrightarrow} y_{1}^{\prime} \cdot \chi_{2} \cdot x_{2} \stackrel{c ? m}{\longrightarrow} x_{2}^{\prime} \cdot \rho_{2}$ Since $\chi_{2}$ contains no move of $q$, the run $\chi_{2} \cdot x_{2} \stackrel{c ? m}{\longrightarrow} x_{2}^{\prime}$ can be reordered into a run of the form $y_{2} \stackrel{c ? m}{\longrightarrow} y_{2}^{\prime} \cdot \chi_{3}$. So the run $\rho$ is causal-equivalent to $\chi_{1} \cdot y_{1} \stackrel{c ! m}{\longrightarrow} y_{1}^{\prime} \cdot y_{2} \stackrel{c ? m}{\longrightarrow} y_{2}^{\prime} \cdot \chi_{3} \cdot \rho_{2}$. Since $\chi_{1}$ contains no move of $p$, the channel $c$ is empty in $y_{2}^{\prime}$. It follows from the induction hypothesis that $\chi_{3} \cdot \rho_{2}$ is causalequivalent to a run that is synchronous for $c$, hence, so does $\rho$.

Proposition 3.4 (Synchronisation). If $c$ is an essential channel in $\mathcal{T}$, then $\operatorname{REACH}(\mathcal{T}) \preceq_{m} \operatorname{REACH}(\mathcal{U})$ where $\mathcal{U}$ results from the synchronisation of $c$ in $\mathcal{T}$.

Proof. We assume that $\mathcal{T}$ and $\mathcal{U}$ are given as in Definition 3.3. Consider an instance of $\operatorname{REACH}(\mathcal{T})$, i.e., a system of communicating processes $\mathcal{R}=\left\langle\mathcal{T},\left\{\mathcal{A}^{p}\right\}_{p \in P}\right\rangle$ We construct a new system $\mathcal{S}=\left\langle\mathcal{U},\left\{\mathcal{B}^{p}\right\}_{p \in P}\right\rangle$ such that $\llbracket \mathcal{R} \rrbracket$ has an accepting run if, and only if, $\llbracket \mathcal{S} \rrbracket$ does. Let $p$ and $q$ denote the source and destination of $c$, respectively. Processes different from $p$ and $q$ are left unchanged, i.e., $\mathcal{B}^{r}=\mathcal{A}^{r}$ for $r \in P \backslash\{p, q\}$. The LTSes $\mathcal{B}^{p}$ and $\mathcal{B}^{q}$ are obtained from $\mathcal{A}^{p}$ and $\mathcal{A}^{q}$, respectively, by the following modifications:

- A transmission transition $s \stackrel{c ! m}{\longrightarrow} t$ in $\mathcal{A}^{p}$ becomes $s \stackrel{c_{m} ! 1}{\longrightarrow} \cdot \stackrel{c_{\text {ack }} ? 1}{\longrightarrow} t$ in $\mathcal{B}^{p}$.

- A reception transition $s \stackrel{c ? m}{\longrightarrow} t$ in $\mathcal{A}^{q}$ becomes $s \stackrel{c_{m} ? 1}{\longrightarrow} \cdot \stackrel{c_{\text {ack }} ! 1}{\longrightarrow} t$ in $\mathcal{B}^{q}$.

- All the other transitions are left unchanged.

It is understood that a new intermediate state is created in $\mathcal{B}^{p}$ and $\mathcal{B}^{q}$ for each modified transition (i.e., for each communication on $c$ ).

We now show that $\mathcal{S}$ faithfully simulates $\mathcal{R}$. Assume that $\llbracket \mathcal{R} \rrbracket$ has an accepting run. By Lemma 3.2, $\llbracket \mathcal{R} \rrbracket$ has an accepting run $\rho$ which is synchronous for $c$. So each communication on $c$ in $\rho$ is of the form $(\boldsymbol{s}, \boldsymbol{v}) \stackrel{c ! m}{\longrightarrow} \cdot \stackrel{c ? m}{\longrightarrow}(\boldsymbol{t}, \boldsymbol{v})$, with $v^{c}=\varepsilon$. By construction, $\mathcal{S}$ can simulate this situation via the following run:

$$
(\boldsymbol{s}, \boldsymbol{w}) \stackrel{c_{m} ! 1}{\longrightarrow} \cdot \stackrel{c_{m} ? 1}{\longrightarrow} \cdot \stackrel{c_{\mathrm{ack}} ! 1}{\longrightarrow} \cdot \stackrel{c_{\mathrm{ack}} ? 1}{\longrightarrow}(\boldsymbol{t}, \boldsymbol{w})
$$


where $\boldsymbol{w}$ is obtained from $\boldsymbol{v}$ in the obvious way: $w^{d}=v^{d}$ for all $d \in(C \backslash\{c\})$ and $w^{d}=\varepsilon$ for all $d \in\left(C^{\prime} \backslash C\right)$. By simulating in this fashion all synchronous communications on $c$ in $\rho$, we obtain an accepting run in $\llbracket \mathcal{S} \rrbracket$.

Conversely, assume that $\llbracket \mathcal{S} \rrbracket$ has an accepting run $\sigma$. By construction of $\mathcal{B}^{p}$ and $\mathcal{B}^{q}$, we may decompose $\sigma$ into a join of runs of the form

$$
\chi_{0} \cdot x_{1} \stackrel{c_{m} ! 1}{\longrightarrow} y_{1} \cdot \chi_{1} \cdot x_{2} \stackrel{c_{m} ? 1}{\longrightarrow} y_{2} \cdot \chi_{2} \cdot x_{3} \stackrel{c_{\text {ack }} ! 1}{\longrightarrow} y_{3} \cdot \chi_{3} \cdot x_{4} \stackrel{c_{\text {ack }} ? 1}{\longrightarrow} y_{4} \cdot \chi_{4}
$$

such that each $\chi_{i}$ contains no communication action on $C^{\prime} \backslash C$. It is readily seen that the channels in $C^{\prime} \backslash C$ are all empty in $x_{1}, y_{2}, x_{3}$ and $y_{4}$. Moreover, $p$ does not move in $\chi_{1}, \chi_{2}$ and $\chi_{3}$, and $q$ does not move in $\chi_{2}$. So we may reorder the run (2) into a causal-equivalent run of the same form such that $\chi_{1}, \chi_{2}$ and $\chi_{3}$ are all of zero length. It follows that $\sigma$ is causal-equivalent to a join $\sigma^{\prime}$ of runs that either contain no communication action on $C^{\prime} \backslash C$, or are of the form (1). By construction, $\mathcal{R}$ can simulate the latter by a run $(\boldsymbol{s}, \boldsymbol{v}) \stackrel{c ! m}{\longrightarrow} \cdot \stackrel{c ? m}{\longrightarrow}(\boldsymbol{t}, \boldsymbol{v})$, where $\boldsymbol{v}$ is obtained from $\boldsymbol{w}$ in the obvious way. By simulating in this fashion all runs of the form (1) in $\sigma^{\prime}$, we obtain an accepting run in $\llbracket \mathcal{R} \rrbracket$.

Proposition 3.10 (Split). If $c$ is an irreversible channel in $\mathcal{T}$, then it holds that $\operatorname{REACH}(\mathcal{T}) \preceq_{m} \operatorname{REACH}(\mathcal{U})$ where $\mathcal{U}$ results from the split of $c$ in $\mathcal{T}$.

Proof. We assume that $\mathcal{T}$ and $\mathcal{U}$ are given as in Definition 3.6. Consider an instance of $\operatorname{REACH}(\mathcal{T})$, i.e., a system of communicating processes $\mathcal{R}=\left\langle\mathcal{T},\left\{\mathcal{A}^{p}\right\}_{p \in P}\right\rangle$. We construct a new system $\mathcal{S}=\left\langle\mathcal{U},\left\{\mathcal{B}^{p}\right\}_{p \in P}\right\rangle$ such that $\llbracket \mathcal{R} \rrbracket$ has an accepting run if, and only if, $\llbracket \mathcal{S} \rrbracket$ does. Let $p$ and $q$ denote the source and destination of $c$, respectively. Processes different from $p$ and $q$ are left unchanged. The LTSes $\mathcal{B}^{p}$ and $\mathcal{B}^{q}$ are the same as $\mathcal{A}^{p}$ and $\mathcal{A}^{q}$, respectively, except that transmissions $c ! m$ of $p$ are replaced by transmissions $c_{0} ! m$, and receptions $c ? m$ of $q$ are replaced by transmissions $c_{1} ! m$. The new process $r$ just matches messages from $c_{0}$ and $c_{1}: \mathcal{B}^{r}$ has a state $s_{\text {idle }}$ which is both initial and final, and one intermediate state $s_{m}$ for each message $m \in \operatorname{msg}(c)$, along with transitions $s_{\text {idle }} \stackrel{c_{0} ? m}{\longrightarrow} s_{m} \stackrel{c_{1} ? m}{\longrightarrow} s_{\text {idle }}$.

It is readily seen that an accepting run in $\llbracket \mathcal{R} \rrbracket$ can be step-wise translated into a run in $\llbracket \mathcal{S} \rrbracket$ that ends with the same contents in $c_{0}$ and $c_{1}$. This latter run can be extended into an accepting one by appending moves of $r$ to match messages in $c_{0}$ and $c_{1}$, until both of them become empty.

Conversely, assume that $\llbracket \mathcal{S} \rrbracket$ has an accepting run. By Lemma 3.8, $\llbracket \mathcal{S} \rrbracket$ has an accepting run $\sigma$ which is causal for $p$. Note that there is no directed path from $q$ to $p$ in $\mathcal{U}$, by irreversibility of $c$ in $\mathcal{T}$. Since $\sigma$ is causal for $p$, each move of $p$ occurs before each move of $q$. So, in $\sigma$, all transmissions on $c_{0}$ occur before any transmission on $c_{1}$. Moreover, the definition of $r$ ensures that the strings received from $c_{0}$ and $c_{1}$ are equal. Therefore, we massage $\sigma$ as follows: we replace transmissions $c_{0} ! m$ of $p$ by $c ! m$, we replace transmissions $c_{1} ! m$ of $q$ by receptions $c ? m$, and we remove the moves of $r$. The result is an accepting run in $\llbracket \mathcal{R} \rrbracket$. 


\section{B Proofs for Section 4}

Distribution of a process via multi-way rendezvous synchronisation. Consider a topology $\mathcal{T}=\langle P, C, \ldots\rangle$ and a non-empty set $D \subseteq C$ of channels in $\mathcal{T}$, and assume that $\mathcal{U}$ results from the fusion of $D$ in $\mathcal{T}$. Let $p_{1}, \ldots, p_{n}$ denote the endpoints of $D$. The set of processes of $\mathcal{U}$ is, by definition, $Q=\left(P \backslash\left\{p_{1}, \ldots, p_{n}\right\}\right) \cup\{u\}$, where $u \notin(P \cup C \cup M)$ corresponds to the merging of $p_{1}, \ldots, p_{n}$. We show that (multi-way) rendezvous synchronisation allows $p_{1}, \ldots, p_{n}$ to coordinate in $\mathcal{T}$ and simulate the behaviour of $u$ in $\mathcal{U}$. Consider a system of communicating processes $\mathcal{S}=\left\langle\mathcal{U},\left\{\mathcal{B}^{q}\right\}_{q \in Q}\right\rangle$. We construct a new system $\mathcal{S}^{\prime}=\left\langle\mathcal{T},\left\{\mathcal{A}^{p}\right\}_{p \in P}\right\rangle$, with rendezvous synchronisation between $p_{1}, \ldots, p_{n}$, such that $\llbracket \mathcal{S} \rrbracket$ has an accepting run if, and only if, $\llbracket \mathcal{S}^{\prime} \rrbracket$ does.

Processes different from $u$ are left unchanged, i.e., $\mathcal{A}^{q}=\mathcal{B}^{q}$ for $q \in Q \backslash\{u\}$. The processes $p_{1}, \ldots, p_{n}$ behave like $u$, except that $p_{i}$ simply internalises the communication actions on channels that it is not connected to. For the simulation to be faithful, we use rendezvous synchronisation to guarantee that $p_{1}, \ldots, p_{n}$ all follow the same "local" run of $u$. To this end, we take the synchronisation alphabet $\Sigma$ to be the set of transitions of $\mathcal{B}^{u}$. Formally, the LTS $\mathcal{A}^{p_{i}}$ is obtained from $\mathcal{B}^{u}$ by the following modifications:

- Communication actions of $\mathcal{B}^{u}$ that are not in $A_{\text {com }}^{p_{i}}$ are replaced by a new internal action skip.

- Each transition $s \stackrel{a}{\longrightarrow} t$ in $\mathcal{B}^{u}$ becomes two transitions $s \stackrel{(s, a, t)}{\longrightarrow} \cdot \stackrel{a_{i}}{\longrightarrow} t$ in $\mathcal{A}^{p_{i}}$, with a new intermediate state in-between, and where $a_{i}$ is defined by $a_{i}=a$ if $a \in A_{\text {com }}^{p_{i}}$ and $a_{i}=$ skip otherwise. The first transition performs a rendezvous synchronisation to ensure that all other $p_{j}$ 's perform the same transition. The second transition makes $p_{i}$ execute $a$ only if $a$ is under $p_{i}$ 's responsibility.

It is easy to see that, by construction, $\llbracket \mathcal{S} \rrbracket$ has an accepting run if, and only if, $\llbracket \mathcal{S}^{\prime} \rrbracket$ has an accepting run.

Restriction to two-message alphabets. While the distribution construction above requires a synchronisation alphabet with many actions in general, we only show in Sec. 4 how to simulate rendezvous synchronisation over just two (abstract) actions $\{a, b\}$. This makes the synchronisation protocol and its correctness proof simpler. In fact, there is no loss of generality, since rendezvous synchronisation over a larger alphabet $\Sigma=\left\{m_{0}, \ldots, m_{k}\right\}$ can be simulated with just a binary alphabet $\{a, b\}$. Indeed, a single rendezvous synchronisation over $m_{i} \in \Sigma$ can be replaced by $i+1$ rendezvous synchronisations $a^{i} b$, where $a$ is used to encode $m_{i}$ in unary, and $b$ is used as a separator. Of course, other encodings are possible.

Proposition 4.3 (Fusion). If $D$ is the support of a closed directed walk in a topology $\mathcal{T}$, then $\operatorname{REACH}(\mathcal{U}) \preceq_{m} \operatorname{REACH}(\mathcal{T})$ where $\mathcal{U}$ results from the fusion of $D$ in $\mathcal{T}$. 
Proof. By induction on the cardinality of $D$. The base case $D=\emptyset$ follows from the observation that the fusion of $\emptyset$ in $\mathcal{T}$ is identical to $\mathcal{T}$ except that it contains one additional process that is not connected to any channel. For the induction step, let $D$ be a non-empty set of channels, and assume that the proposition ("if ...then ...") holds for all proper subsets of $D$. Denote by $\mathcal{U}$ the fusion of $D$ in $\mathcal{T}$. By definition, there exists a closed directed walk $p_{0} \stackrel{c_{1}}{\Longrightarrow} p_{1} \stackrel{c_{2}}{\Longrightarrow} \cdots \stackrel{c_{n}}{\Longrightarrow} p_{n}=p_{0}$ in $\mathcal{T}$ whose support is $D=\left\{c_{1}, \ldots, c_{n}\right\}$. If this directed walk is also a directed cycle, i.e., $p_{1}, \ldots, p_{n}$ are pairwise distinct, then we conclude thanks to Lemma 4.2. Otherwise, there exist $1 \leq k \leq l \leq n$ such that $p_{k-1} \stackrel{c_{k}}{\Longrightarrow} p_{k} \stackrel{c_{k+1}}{\Longrightarrow} \cdots \stackrel{c_{l}}{\Longrightarrow} p_{l}$ is a directed cycle. By Lemma 4.2 , it holds that $\operatorname{REACH}(\hat{\mathcal{T}}) \preceq_{m} \operatorname{REACH}(\mathcal{T})$ where $\hat{\mathcal{T}}$ results from the fusion of $\left\{c_{k}, \ldots, c_{l}\right\}$ in $\mathcal{T}$. Denote by $\hat{p}$ the process in $\hat{\mathcal{T}}$ that corresponds to the merging of all endpoints of $\left\{c_{k}, \ldots, c_{l}\right\}$. Let $\hat{D}=D \backslash\left\{c_{k}, \ldots, c_{l}\right\}$. The closed directed walk $p_{0} \stackrel{c_{1}}{\Longrightarrow} p_{1} \stackrel{c_{2}}{\Longrightarrow} \cdots \stackrel{c_{n}}{\Longrightarrow} p_{n}=p_{0}$ of $\mathcal{T}$ gives rise to a closed directed walk in $\hat{\mathcal{T}}$ with support $\hat{D}$. Indeed, the latter is obtained by collapsing, into $\hat{p}$, all $p_{i-1} \stackrel{c_{i}}{\Longrightarrow} p_{i}$ with $1 \leq i \leq n$ and $c_{i} \in\left\{c_{k}, \ldots, c_{l}\right\}$. Observe that $\mathcal{U}$ also results from the fusion of $\hat{D}$ in $\hat{\mathcal{T}}$. Since $\hat{D}$ is a proper subset of $D$, we derive from the induction hypothesis that $\operatorname{REACH}(\mathcal{U}) \preceq_{m} \operatorname{REACH}(\hat{\mathcal{T}}) \preceq_{m} \operatorname{REACH}(\mathcal{T})$, which concludes the proof of the proposition.

\section{Proofs for Section 5}

\section{C.1 Technical Details for Subsection 5.1}

Recall that $\xi=\left(p_{0}, c_{1}, q_{1}, p_{1}, \ldots, c_{n}, q_{n}, p_{n}\right)$ is a jumping cycle in $\mathcal{T}$, and that

$$
p_{0} \stackrel{c_{1}}{=} q_{1} \approx_{D_{1}} p_{1} \cdots \stackrel{c_{n}}{=} q_{n} \approx_{D_{n}} p_{n}
$$

for some pairwise disjoint subsets $D_{1}, \ldots, D_{n}$ of $C \backslash\left\{c_{1}, \ldots, c_{n}\right\}$ that are each the support of a closed directed walk in $\mathcal{T}$. Recall also that $\mathcal{U}_{0}=\mathcal{T}$ and that $\mathcal{U}_{i}$ results from the fusion of $D_{i}$ in $\mathcal{U}_{i-1}$, for each $1 \leq i \leq n$. It follows from Definition 4.1 that $\mathcal{U}_{i}=\left\langle P_{i}, C_{i}, M, \operatorname{src}_{i}\right.$, dst ${ }_{i}$, msg $\left._{i}\right\rangle$ satisfies:

$$
\begin{aligned}
P_{i} & =\left(P_{i-1} \backslash\left\{\operatorname{src}(c), \operatorname{dst}(c) \mid c \in D_{i}\right\}\right) \cup\left\{u_{i}\right\} \\
C_{i} & =C_{i-1} \backslash D_{i} \\
\operatorname{src}_{i}(c) & =f_{i}\left(\operatorname{src}_{i-1}(c)\right) \\
\operatorname{dst}_{i}(c) & =f_{i}\left(\operatorname{dst}_{i-1}(c)\right) \\
\operatorname{msg}_{i}(c) & =\operatorname{msg}_{i-1}(c)
\end{aligned}
$$

where $u_{i}$ is a new process that is not in $P_{i-1} \cup C_{i-1} \cup M_{i-1}$, and $f_{i}: P_{i-1} \rightarrow P_{i}$ is defined by $f_{i}(p)=p$ if $p \in P_{i}$ and $f_{i}(p)=u_{i}$ otherwise. Put differently, the function $f_{i}$ maps each process $p$ in $P_{i-1}$ to either $u_{i}$ if $p$ is among the endpoints of $D_{i}$, or $p$ otherwise. Let us introduce the functions $g_{0}, \ldots, g_{n}$ defined by $g_{i}=f_{i} \circ \cdots \circ f_{1}$. It is understood that $g_{0}$ is the identity function on $P$. 
It is readily seen that $C_{i}=C \backslash\left(D_{1} \cup \cdots \cup D_{i}\right)$ for every $1 \leq i \leq n$. Moreover, $\operatorname{src}_{i}(c)=g_{i}(\operatorname{src}(c))$ and $\mathrm{dst}_{i}(c)=g_{i}(\operatorname{dst}(c))$ for all $c \in C_{i}$. It follows that for every processes $p, q \in P$ and for every channel $c \in C_{i}$, if $p \stackrel{c}{\Longrightarrow} q$ in $\mathcal{T}$, then $g_{i}(p) \stackrel{c}{\Longrightarrow} g_{i}(q)$ in $\mathcal{U}_{i}$. We derive the following property.

Claim. For every $0 \leq i<j \leq n$, there exists in $\mathcal{U}_{i}$ a closed directed walk whose support is $D_{j}$ and that visits $g_{i}\left(q_{j}\right)$ and $g_{i}\left(p_{j}\right)$.

Proof. Let $0 \leq i<j \leq n$. Recall that $D_{j}$ is the support of a closed directed walk $\pi$ in $\mathcal{T}$. Since $q_{j} \approx_{D_{j}} p_{j}$ in $\mathcal{T}, \pi$ necessarily visits both $q_{j}$ and $p_{j}$. Observe that $D_{j} \subseteq C_{i}$. So by applying $g_{i}$ to each process in $\pi$, we obtain a closed directed walk in $\mathcal{U}_{i}$ whose support is $D_{j}$ and that visits $g_{i}\left(q_{j}\right)$ and $g_{i}\left(p_{j}\right)$, which concludes the proof of the claim.

This property entails the two following facts, that are used without proof in Subsec. 5.1.

- For all $1 \leq i \leq n$, the set $D_{i}$ is the support of a closed directed walk in $\mathcal{U}_{i-1}$.

$-\xi$ leads to a closed undirected walk $g_{n}\left(p_{0}\right) \stackrel{c_{1}}{=} g_{n}\left(p_{1}\right) \ldots \stackrel{c_{n}}{=} g_{n}\left(p_{n}\right)$ in $\mathcal{U}_{n}$.

This follows from the observation that, for all $1 \leq i \leq n$,

- $C_{n}=C \backslash\left(D_{1} \cup \cdots \cup D_{n}\right)$ and each $D_{j}$ is contained in $C \backslash\left\{c_{1}, \ldots, c_{n}\right\}$, hence, $c_{i} \in C_{n}$.

- $g_{i-1}\left(q_{i}\right)$ and $g_{i-1}\left(p_{i}\right)$ are among the endpoints of $D_{i}$ in $\mathcal{U}_{i-1}$, which entails that $g_{i}\left(q_{i}\right)=u_{i}=g_{i}\left(p_{i}\right)$, hence, $g_{n}\left(q_{i}\right)=g_{n}\left(p_{i}\right)$.

\section{C.2 Proof of Lemma 5.4}

Lemma 5.4. Consider a topology $\mathcal{T}$ and an essential non-unary channel $c$ therein. Let $\mathcal{U}$ be the topology obtained from $\mathcal{T}$ by adding an acknowledgement channel for $c$. Then $\mathcal{T}$ contains a jumping cycle if $\mathcal{U}$ contains a jumping cycle.

Proof. We first fix some notations. Assume that $\mathcal{T}=\langle P, C, M$, src, dst, msg $\rangle$. Consider the topology $\mathcal{U}=\left\langle P, C^{\prime}, M, \mathrm{src}^{\prime}, \mathrm{dst}^{\prime}, \mathrm{msg}^{\prime}\right\rangle$ defined by $C^{\prime}=C \cup\{\overleftarrow{c}\}$ and

$$
\left(\operatorname{src}^{\prime}(d), \operatorname{dst}^{\prime}(d), \operatorname{msg}^{\prime}(d)\right)= \begin{cases}(\operatorname{src}(d), \operatorname{dst}(d), \operatorname{msg}(d)) & \text { if } d \in C \\ (\operatorname{dst}(c), \operatorname{src}(c),\{1\}) & \text { if } d=\overleftarrow{c}\end{cases}
$$

where $\overleftarrow{c}$ is a new channel that is not in $P \cup C \cup M$. Suppose that $\mathcal{U}$ contains a jumping cycle $\left(p_{0}, c_{1}, q_{1}, p_{1}, \ldots, c_{n}, q_{n}, p_{n}\right)$. It follows from Remark 5.2 that, in the topology $\mathcal{U}$,

$$
p_{0} \stackrel{c_{1}}{=} q_{1} \approx_{D_{1}} p_{1} \cdots \stackrel{c_{n}}{=} q_{n} \approx_{D_{n}} p_{n}=p_{0}
$$

for some pairwise disjoint subsets $D_{1}, \ldots, D_{n}$ of $C^{\prime} \backslash\left\{c_{1}, \ldots, c_{n}\right\}$. By Remark 5.1, it is enough to show that $\mathcal{T}$ contains a jumping circuit. Observe that $c_{i} \neq \overleftarrow{c}$ for all $1 \leq i \leq n$, since $\overleftarrow{c}$ is a unary channel. If $\overleftarrow{c}$ is not in $D_{1} \cup \cdots \cup D_{n}$, then $\left(p_{0}, c_{1}, q_{1}, p_{1}, \ldots, c_{n}, q_{n}, p_{n}\right)$ is a jumping circuit in $\mathcal{T}$, and we are done. Otherwise, 
$\overleftarrow{c}$ is contained in exactly one of the sets $D_{i}$. We may assume, w.l.o.g., that the set $D_{i}$ containing $\overleftarrow{c}$ is $D_{1}$. If $q_{1}$ and $p_{1}$ are synchronizable over $D_{1} \backslash\{\overleftarrow{c}\}$ then, again, $\left(p_{0}, c_{1}, q_{1}, p_{1}, \ldots, c_{n}, q_{n}, p_{n}\right)$ is a jumping circuit in $\mathcal{T}$, and we are done. So we suppose, for the remainder of the proof, that $q_{1}$ and $p_{1}$ are not synchronizable over $D_{1} \backslash\{\overleftarrow{c}\}$. Note that this entails, in particular, that $p_{1} \neq q_{1}$ Define $E_{1}=D_{1} \backslash\{c, \overleftarrow{c}\}$. The existence of a jumping circuit in $\mathcal{T}$ will follow from the two following properties:

$$
c \in D_{1}
$$

$$
\left(p \approx_{E_{1}} p_{1} \wedge q \approx_{E_{1}} q_{1}\right) \quad \text { or } \quad\left(p \approx_{E_{1}} q_{1} \wedge q \approx_{E_{1}} p_{1}\right)
$$

where $p$ and $q$ are the source and destination of $c$, respectively. Let us prove these properties. Since $q_{1} \approx_{D_{1}} p_{1}$ in $\mathcal{U}$, there exists a directed path $\pi$ from $p_{1}$ to $q_{1}$, and a directed path $\chi$ from $q_{1}$ to $p_{1}$, such that both $\pi$ and $\chi$ use only channels in $D_{1}$. Moreover, at least one of them contains $\overleftarrow{c}$, since $q_{1}$ and $p_{1}$ are not synchronizable over $D_{1} \backslash\{\overleftarrow{c}\}$. The concatenation $\pi \cdot \chi$ is a closed directed walk containing $\overleftarrow{c}$. By essentiality of $c$, this closed directed walk necessarily contains $c$ (since $\overleftarrow{c}$ has source $q$ and destination $p$ ). We have shown that $\pi \cdot \chi$ contains both $c$ and $\overleftarrow{c}$. We derive that $c \in D_{1}$, i.e., that (3) holds. Moreover, since $\pi$ and $\chi$ are open ${ }^{6}$ directed paths, we obtain that:

- Either $\pi$ contains exactly one occurrence of $c$ and no occurrence of $\overleftarrow{c}$, and $\chi$ contains exactly one occurrence of $\overleftarrow{c}$ and no occurrence of $c$. So we may decompose $\pi$ and $\chi$ into

$$
\pi=\pi^{\prime} \cdot p \stackrel{c}{\Longrightarrow} q \cdot \pi^{\prime \prime} \quad \text { and } \quad \chi=\chi^{\prime} \cdot q \stackrel{\overleftarrow{c}}{\Longrightarrow} p \cdot \chi^{\prime \prime}
$$

where $\pi^{\prime}, \pi^{\prime \prime}, \chi^{\prime}$ and $\chi^{\prime \prime}$ are directed paths that neither contain $c$ nor $\overleftarrow{c}$, i.e., that use only channels in $E_{1}$. The paths $\pi^{\prime}$ and $\chi^{\prime \prime}$ show that $p \approx_{E_{1}} p_{1}$, and the paths $\pi^{\prime \prime}$ and $\chi^{\prime}$ show that $q \approx_{E_{1}} q_{1}$.

- Or $\pi$ contains exactly one occurrence of $\overleftarrow{c}$ and no occurrence of $c$, and $\chi$ contains exactly one occurrence of $c$ and no occurrence of $\overleftarrow{c}$. We obtain, with the same arguments as in the previous case, that $p \approx_{E_{1}} q_{1}$ and $q \approx_{E_{1}} p_{1}$.

We obtain in both cases that (4) holds.

Let us exploit the properties (3) and (4) to transform the jumping cycle $\left(p_{0}, c_{1}, q_{1}, p_{1}, \ldots, c_{n}, q_{n}, p_{n}\right)$ into a jumping circuit that does not use $\overleftarrow{c}$. Recall that $\overleftarrow{c} \notin\left\{c_{1}, \ldots, c_{n}\right\} \cup D_{2} \cup \cdots \cup D_{n}$. So $\overleftarrow{c}$ is only used in $q_{1} \approx_{D_{1}} p_{1}$. Expand in the jumping cycle, $\left(q_{1}, p_{1}\right)$ into $\left(q_{1}, p, c, q, p_{1}\right)$ or $\left(q_{1}, q, c, p, p_{1}\right)$, depending on which disjunct of (4) holds. The resulting sequence is a jumping circuit in $\mathcal{T}$. Indeed, it follows from (3) that $c$ was not already among $c_{1}, \ldots, c_{n}$, and that $c \notin D_{i}$ for all $2 \leq i \leq n$. This concludes the proof of the lemma.

\footnotetext{
${ }^{6}$ A directed walk is called open when it is not closed, i.e., when it starts and ends in distinct processes.
} 


\section{C.3 Proofs of Corollary 5.5, Lemma 5.7 and Lemma 5.8}

Corollary 5.5. Consider a topology $\mathcal{T}$ and an essential non-unary channel $c$ therein. Let $\mathcal{U}$ be the topology resulting from the synchronisation of $c$ in $\mathcal{T}$. Then $\mathcal{T}$ contains a jumping cycle if $\mathcal{U}$ contains a jumping cycle.

Proof. Assume that $\mathcal{U}$ contains a jumping cycle $\left(p_{0}, c_{1}, q_{1}, p_{1}, \ldots, c_{n}, q_{n}, p_{n}\right)$. Let $\mathcal{V}$ be the topology obtained from $\mathcal{T}$ by adding an acknowledgement channel $\overleftarrow{c}$ for $c$. It is readily seen that $\left(p_{0}, c_{1}, q_{1}, p_{1}, \ldots, c_{n}, q_{n}, p_{n}\right)$ is also a jumping cycle in $\mathcal{V}$. It follows from Lemma 5.4 that $\mathcal{T}$ contains a jumping cycle.

Lemma 5.7. Consider a topology $\mathcal{T}$ and a non-unary channel $c$ therein. If $\mathcal{T}$ is divided, then so is the topology resulting from the synchronisation of $c$ in $\mathcal{T}$.

Proof. We assume that $\mathcal{T}$ and $\mathcal{U}$ are given as in Definition 3.3. Consider an irreversible unary channel $b$ in $\mathcal{U}$. Observe that no channel in $C^{\prime} \backslash C$ is irreversible. It follows that $b$ is also a unary channel in $\mathcal{T}$. Furthermore, its destination is the same process in $\mathcal{T}$ and $\mathcal{U}$, say $r$. It is routinely checked that $b$ is also irreversible in $\mathcal{T}$. Since $\mathcal{T}$ is divided and $c$ is non-unary, we obtain that $r$ is neither the source nor destination of $c$. Moreover, in $\mathcal{T}, r$ is a sink and is not the destination of some non-unary channel. It follows that these two properties also hold in $\mathcal{U}$.

Lemma 5.8. If $\mathcal{T}$ is a divided topology with no jumping cycle, then every nonunary channel in $\mathcal{T}$ is essential.

Proof. Consider a non-unary channel $c$ in $\mathcal{T}$, with source $p$ and destination $q$. Assume, by contradiction, that $c$ is not essential. This means that there exists a directed path $\pi$ from $p$ to $q$ that does not contain $c$.

We first show that $c$ is irreversible. If $q \stackrel{*}{\Longrightarrow} p$, then there is a directed path from $q$ to $p$ that does not contain $c$. It follows that $(p, c, q, p)$ is a jumping cycle in $\mathcal{T}$, which contradicts the assumption that $\mathcal{T}$ contains no jumping cycle.

Let us decompose $\pi$ into a join of directed paths that either remain in the same strongly-connected component, or contain a single irreversible channel (i.e., a channel between two different strongly-connected components). Note that $\pi$ contains at least one irreversible channel since there is no directed path from $q$ to $p$. Formally,

$$
\pi=\chi_{0} \cdot p_{0} \stackrel{c_{1}}{\Longrightarrow} q_{1} \cdot \chi_{1} \cdots p_{n-1} \stackrel{c_{n}}{\Longrightarrow} q_{n} \cdot \chi_{n}
$$

where $n \geq 1$, each $c_{i}$ is an irreversible channel, and each $\chi_{i}$ is a directed path containing only reversible channels. Since $\mathcal{T}$ is divided, the channels $c_{1}, \ldots, c_{n}$ are necessarily non-unary. Indeed, if $c_{i}$ is unary, then $q_{i}$ is a sink, hence $i=n$ and $\chi_{n}$ has length zero. This entails that $q_{i}=q$ is the destination of the nonunary channel $c$, which contradicts the assumption that $\mathcal{T}$ is divided. Define $q_{0}=p$ and $p_{n}=q$. Each directed path $\chi_{i}$ starts in $q_{i}$ and ends in $p_{i}$. Recall that all channels in $\chi_{i}$ are reversible. Hence, there exists a directed path $\chi_{i}^{\prime}$ from $p_{i}$ to $q_{i}$, and $\chi_{i}^{\prime}$ also contains only reversible channels. We derive that 
$q_{i} \approx_{D} p_{i}$ for all $0 \leq i \leq n$, where $D=C \backslash\left\{c, c_{1}, \ldots, c_{n}\right\}$. Consider the sequence $\xi=\left(p_{0}, c_{1}, q_{1}, p_{1}, \ldots, c_{n}, q_{n}, p_{n}, c, p, p_{0}\right)$. The channels $c, c_{1}, \ldots, c_{n}$ are pairwise distinct since $\pi$ is a directed path that does not contain $c$. We obtain that $\xi$ is a jumping circuit, which, together with Remark 5.1, contradicts the assumption that $\mathcal{T}$ contains no jumping cycle.

\section{C.4 On Remark 5.6}

The following fact is not used to prove Theorem 5.3, but it was mentioned in Remark 5.6.

Lemma C.1. Consider a topology $\mathcal{T}$ and an essential non-unary channel $c$ therein. Let $\mathcal{U}$ be the topology resulting from the synchronisation of $c$ in $\mathcal{T}$. Then $\mathcal{T}$ contains a jumping cycle only if $\mathcal{U}$ contains a jumping cycle.

Proof. We assume that $\mathcal{T}$ and $\mathcal{U}$ are given as in Definition 3.3. Suppose that $\mathcal{T}$ contains a jumping cycle

$$
p_{0} \stackrel{c_{1}}{=} q_{1} \approx_{D} p_{1} \ldots \stackrel{c_{n}}{=} q_{n} \approx_{D} p_{n}=p_{0}
$$

where $D=C \backslash\left\{c_{1}, \ldots, c_{n}\right\}$. By Remark 5.1, it is enough to show that $\mathcal{U}$ contains a jumping circuit. Let us define $D^{\prime}=C^{\prime} \backslash\left\{c_{1}, \ldots, c_{n}\right\}$. Observe that $D^{\prime}=(D \backslash\{c\}) \cup\left\{c_{m} \mid m \in \operatorname{msg}(c)\right\} \cup\left\{c_{\text {ack }}\right\}$. We derive that $\approx_{D^{\prime}}$ contains $\approx_{D}$. Hence, $q_{i} \approx_{D^{\prime}} p_{i}$ for every $1 \leq i \leq n$. If $c \notin\left\{c_{1}, \ldots, c_{n}\right\}$ then we get that $\left(p_{0}, c_{1}, q_{1}, p_{1}, \ldots, c_{n}, q_{n}, p_{n}\right)$ is a jumping circuit in $\mathcal{U}$, and we are done. Otherwise, $c \in\left\{c_{1}, \ldots, c_{n}\right\}$. We may assume, w.l.o.g., that $c_{n}=c$. Observe that $c_{1}, \ldots, c_{n-1}$ are pairwise distinct non-unary channels in $\mathcal{U}$. Moreover, it holds that $p_{n-1} \approx_{D^{\prime}} q_{n}$ since $c_{n}=c$. By transitivity of $\approx_{D^{\prime}}$, we obtain that $q_{n-1} \approx_{D^{\prime}} p_{n}$. So we may remove $p_{n-1} \stackrel{c_{n}}{=} q_{n}$ from the sequence. To prove that the resulting sequence $\left(p_{0}, c_{1}, q_{1}, p_{1}, \ldots, c_{n-1}, q_{n-1}, p_{n}\right)$ is a jumping cycle in $\mathcal{U}$, we only need to show that $n \geq 2$. By contradiction, suppose that $n=1$. This means that $\left(p_{0}, c, q_{1}, p_{1}\right)$ is a jumping cycle in $\mathcal{T}$, It follows that $p \approx_{D} q$ and $c \notin D$. Hence, there exists a directed path from $p$ to $q$ using only channels in $D$, which contradicts the assumption that $c$ is essential.

\section{C.5 Proof of Theorem 5.9}

Theorem 5.9. REACHNJC is equivalent to reachability in Petri nets under polynomial-time many-one reductions.

Proof. Let $\mathcal{S}=\left\langle\mathcal{T},\left\{\mathcal{A}^{p}\right\}_{p \in P}\right\rangle$ be a system of communicating processes over a decidable topology $\mathcal{T}=\langle P, C, M$, src, dst, msg $\rangle$. We reduce the reachability problem in $\mathcal{S}$ to the reachability problem of a Petri net of size polynomial in $|\mathcal{S}|$.

Recall that our decidability argument involves a series of polynomial manyone reductions from the original topology $\mathcal{T}$ comprising unary and non-unary channels into a final topology $\mathcal{U}$ containing only unary channels. 
The first reduction step involves splitting irreversible unary channels in $\mathcal{T}$. Every such channel $c$ is replaced by two unary channels $c_{1}$ and $c_{2}$. A new process is added to match the contents of $c_{1}$ and $c_{2}$. We thus obtain a new topology with at most $|C|$ extra processes and $|C|$ extra channels. The system $\mathcal{S}$ is transformed into another system $\mathcal{S}_{0}$ where the extra processes have 2 states and 2 transitions each, i.e., constant size. Thus, $\left|\mathcal{S}_{0}\right|=|\mathcal{S}|+O(|C|)=O(|\mathcal{S}|)$.

All the subsequent reduction steps consist of making (essential) non-unary channels synchronous by replacing them with unary channels (see Sec. 3). More precisely, each reduction step replaces a non-unary channel $p \stackrel{c}{\Longrightarrow} q$ by $|\operatorname{msg}(c)|$ unary channels from $p$ to $q$, plus one unary channel back from $q$ to $p$. Thus, the number of channels in the topology increases by $\sum_{c \in C}|\operatorname{msg}(c)|$. Regarding the alphabet size, since the original channel $c$ is replaced by $|\operatorname{msg}(c)|+1$ unary channels, the total alphabet size is increased just by $O(|C|)$. Consequently, the size of the topology is increased by $O\left(|C|+\sum_{c \in C}|\operatorname{msg}(c)|\right)$.

In the resulting topology, every transmission of message $m$ by process $p$ on channel $c$ is replaced by a transmission on the corresponding unary channel $c_{m}$, followed by a reception of an acknowledgement from $q$ on the back channel. Thus, each communication transition on a non-unary channel $c$ in $\mathcal{S}_{0}$ results in two transitions and an intermediate state in the new system $\mathcal{S}^{\prime}$. Since each transition in $\mathcal{S}_{0}$ is replaced at most once, the size of $\mathcal{S}_{0}$ is increased by at most $2 \cdot \sum_{p \in P}\left|\rightarrow^{p}\right|$. In the end, we obtain: $\left|\mathcal{S}^{\prime}\right|=\left|\mathcal{S}_{0}\right|+O\left(|C|+\sum_{c \in C}|\operatorname{msg}(c)|\right)+O\left(\sum_{p \in P}\left|\rightarrow^{p}\right|\right)=$ $O(|\mathcal{S}|)$.

Clearly, unary channels can be interpreted as counters. Thus system $\mathcal{S}^{\prime}$ can be interpreted as a counter machine (or equivalently a Petri net) with at most $2|C|+\sum_{c \in C}(|\operatorname{msg}(c)|+1)$ counters (split channels + synchronised channels) whose control is obtained by taking the disjoint union of the controls of at most $|P|+|C|$ processes. Notice that this does not require to compute the product of the processes. Thus the counter machine has size linear in $\left|\mathcal{S}^{\prime}\right|$. Clearly, the counter machine has an accepting run iff $\llbracket \mathcal{S}^{\prime} \rrbracket$ has an accepting run iff $\llbracket \mathcal{S} \rrbracket$ has an accepting run. 